Granja-Fernández, R., Maya-Alvarado, B., Cupul-Magaña, A.L., Rodríguez-Troncoso, A.P., Solís-Marín, F.A., \& Sotelo-Casas, R.C. (2021). Echinoderms (Echinodermata) from the Central Mexican Pacific. Revista de Biología Tropical, 69(S1), 219-253. DOI 10.15517/rbt.v69iSuppl.1.46356

DOI 10.15517/rbt.v69iSuppl.1.46356

\title{
Echinoderms (Echinodermata) from the Central Mexican Pacific
}

\author{
Rebeca Granja-Fernández*1 \\ Brenda Maya-Alvarado ${ }^{2}$ \\ Amílcar L. Cupul-Magaña ${ }^{1}$ \\ A. Paola Rodríguez-Troncoso ${ }^{1}$ \\ Francisco A. Solís-Marín ${ }^{3}$ \\ Rosa C. Sotelo-Casas ${ }^{4}$
}

1. Laboratorio de Ecología Marina. Centro Universitario de la Costa, Universidad de Guadalajara. Av. Universidad, Delegación Ixtapa. Puerto Vallarta, México; beckygranja@gmail.com (*Correspondence), amilcar.cupul@gmail.com, pao.rodriguezt@gmail.com

2. Programa de Maestría en Ciencias en Biosistemática y Manejo de Recursos Naturales y Agrícolas, Centro Universitario de Ciencias Biológicas y Agropecuarias, Universidad de Guadalajara, Av. Ing. Ramón Padilla Sánchez, Zapopan, Jalisco, México; brenda.maya9471@alumnos.udg.mx

3. Colección Nacional de Equinodermos “Ma. E. Caso Muñoz”, Laboratorio de Sistemática y Ecología de Equinodermos. Instituto de Ciencias del Mar y Limnología, Universidad Nacional Autónoma de México, Ciudad de México, México; fasolis@cmarl.unam.mx

4. Unidad Multidisciplinaria de Docencia e Investigación Sisal Facultad de Ciencias, Universidad Nacional Autónoma de México. Puerto de Abrigo S/N, Sisal, Yucatán, México; rosacarmensotelocasas@gmail.com

$$
\text { Received 30-XVI-2020. C Corrected 18-VIII-2020. Accepted 19-X-2020. }
$$

\begin{abstract}
Introduction: The echinoderms from the Central Mexican Pacific are of high scientific interest and, prior to this present work, there was a lack of basic information that included incomplete checklists with inconsistencies in systematics and spatial distribution. Objective: To provide a historical review, and an updated checklist with a more complete richness of echinoderms for each state and island of the region. Methods: A checklist was elaborated based on an exhaustive literature search of the Echinodermata, and was complemented with taxonomical revisions of Ophiuroidea scientific collections. All the geographical coordinates of the records were validated. Results: The region harbors 187 species of Echinodermata: three Crinoidea, 35 Asteroidea, 67 Ophiuroidea, 32 Echinoidea, and 50 Holothuroidea. We detected 52 records in the literature that must be considered as invalid and five as doubtful. We provide 16 new records of Ophiuroidea from different states and islands; of them, four are new records for the region. Jalisco presented the highest number of species (84), followed by the coast of Nayarit (74), Michoacán (63), and Colima (55); among the islands, Revillagigedo showed the major number of species (85) followed by Marías (81), Marietas (48), and Isabel (44). Conclusions: The numbers of species known in the region are mostly related to both sampling effort and environmental characteristics that promote high biodiversity. The Central Mexican Pacific is an oceanographic region with mixed conditions from the North and South of the Mexican Pacific, and therefore, with a biogeographical importance reflected in its species richness.
\end{abstract}

Key words: Echinodermata; biodiversity; checklist; historical review; new records. 
The Central Mexican Pacific (CMP) region comprises the states of Nayarit, Jalisco, Colima and Michoacán as well as their coastal (Isabel and Marietas) and oceanic islands (Marías and Revillagigedo). The region is characterized by numerous geographic features such as bays, archipelagos and islands, as well as a heterogenic bottom that combines hard and soft components. The CMP is located in the Tropical Eastern Pacific Province nearby to the Warm Temperate Northeast Pacific Province (Spalding et al., 2007) and for this reason, it shelters northern and southern fauna of the Mexican Pacific (Arriaga-Cabrera et al., 1998). Due to its relevant flora and fauna, most of the islands of the CMP are catalogued as Natural Protected Areas (CONANP, 2005; CONANP, 2007a; CONANP, 2007b; CONANP, 2019).

The study regarding the Echinodermata in the CMP began in 1840 when Gray (1840) reported the asteroids Astropecten regalis and Luidia columbia in Nayarit. Until 1891, efforts in the collection of echinoderms mostly from deep-waters were performed by foreign expeditions such as Albatross and Challenger. As a result, many new records and new species of Crinoidea (Hartlaub, 1895), Asteroidea (Sladen, 1889), Ophiuroidea (Lütken \& Mortensen, 1899), Echinoidea (Agassiz, 1872; Agassiz,1898), and Holothuroidea (Ludwig, 1894) were described.

The $20^{\text {th }}$ century was very productive in terms of the number of published works (75). During this period, numerous foreign expeditions (Albatross, Velero, and Zaca) collected samples from shallow and deep-waters along the region resulting in new records, new species and extensive taxonomic monographs (e.g. Ludwig, 1905; Fisher, 1928a; Fisher, 1928b; Deichmann, 1937; Ziesenhenne, 1937; Ziesenhenne, 1940; Mortensen, 1948a; Mortensen, 1948b). The first national work regarding echinoderms from the CMP was the master's thesis of Caso (1943) who worked with the Asteroidea from México. Caso published dissertations and numerous manuscripts regarding taxonomy (Caso, 1948a; Caso, 1951; Caso, 1961; Caso, 1962a; Caso, 1965; Caso, 1966;
Caso, 1974a; Caso, 1974b; Caso, 1978b; Caso, 1980; Caso,1983b), including descriptions of new species (Caso, 1954; Caso, 1983a; Caso, 1986a), and general accounts on the biodiversity of the CMP (e.g. Caso, 1948b; Caso, 1962b). Their findings helped to better understand the echinoderm fauna from shallow-waters of the region and inspired other Mexican researchers to work with echinoderms from Revillagigedo Islands (Villalobos, 1992; Chacón-Torres et al., 1993; Bautista-Romero, Reyes-Bonilla, LluchCota, \& Lluch-Cota, 1994), Marietas Islands (Nepote-González, 1998), Colima (EspinoBarr, Cibrián-Rodríguez, \& García-Boa, 1996) and even, with species collected in several areas from the CMP that resulted as new taxa (Solís-Marín \& Laguarda-Figueras, 1999). At the end of the $20^{\text {th }}$ century, the first ecological studies in the CMP were published (HolguinQuiñones, 1994; Reyes-Bonilla, 1995; EspinoBarr et al., 1996).

The first two decades of the $21^{\text {st }}$ century have produced a vast number of works (55) regarding topics such as new distribution records (Frontana-Uribe, Hernández-Alcántara, \& Solís-Weiss, 2000; Sotelo-Casas, CupulMagaña, Solís-Marín, \& Rodríguez-Troncoso, 2015; Valdés de Anda, Hendrickx, \& SolísMarín, 2018), description of new species (Honey-Escandón, Solís-Marín, \& LaguardaFigueras, 2011; Solís-Marín, Arriaga-Ochoa, Galván-Villa, \& Laguarda-Figueras, 2018), ecology (Holguin-Quiñones \& Michel-Morfin, 2006; López-Uriarte et al., 2009; HermosilloNuñez et al., 2015; Herrero-Pérezrul, RamírezOrtiz, Rosales-Estrada, \& Reyes-Bonilla, 2015; Hermosillo-Nuñez, Rodríguez-Zaragoza, Ortiz, Calderon-Aguilera, \& Cupul-Magaña, 2016; Sotelo-Casas, Cupul-Magaña, Rodríguez-Zaragoza, Solís-Marín, \& Rodríguez-Troncoso, 2018), and reproduction (Sotelo-Casas, CupulMagaña, Solís-Marín, \& Rodríguez-Troncoso, 2016). It is important to emphasize that most of these studies are focused in the compilation of species of echinoderms inhabiting in different areas of the CMP (Chávez-Dagostino, Nepote-González, Solís-Marín, \& MedinaRosas, 2000; Fuentes-Farías, Villarroel-Melo, 
\& Solís-Marín, 2005; Honey-Escandón, SolísMarín, \& Laguarda-Figueras, 2008; Ríos-Jara, Galván-Villa, \& Solís-Marín, 2008a; Ríos-Jara et al., 2008b; Santos-Beltrán \& Salazar-Silva, 2011; Ríos-Jara et al., 2013; Rodríguez-Troncoso et al., 2013; Solís-Marín et al., 2013a; Granja-Fernández, Herrero-Pérezrul, LópezPérez, Hernández-Morales, \& Rangel-Solís, 2015a; Solís-Marín, Laguarda-Figueras, \& Durán-González, 2016b; Granja-Fernández et al., 2017; Padilla-Pérez, Rodríguez-Troncoso, Sotelo-Casas, \& Cupul-Magaña, 2017; RíosJara, Juárez-Carrillo, \& Galván-Villa, 2017; Nava-Bravo et al., 2019).

Despite the above, the records have shown many inconsistencies such as: 1) the names of some species have been mentioned (written or registered) incorrectly across time (e.g. misspelling), 2) many of the recorded species for the CMP are invalid, and 3) the generated checklists are not complete because they are based only in museum records or material collected mostly in shallow-waters. Thus, the aim of the present manuscript is to provide an accurate and updated checklist of the valid names and synonyms of the Echinodermata from the CMP according to literature, including new distribution records of Ophiuroidea. Moreover, we provide a historical review of the study of each class of Echinodermata, as well as an updated number of species for each state and island in the region.

\section{MATERIALS AND METHODS}

An exhaustive search of published literature for the CMP regarding the classes Crinoidea, Asteroidea, Ophiuroidea, Echinoidea, and Holothuroidea was performed (records dating from 1840 to 2019). Moreover, we visited reference collections in order to taxonomically identify specimens of Ophiuroidea: ICML-UNAM: Colección de Equinodermos "Dra. Ma. Elena Caso", Instituto de Ciencias del Mar y Limnología, Universidad Nacional Autónoma de México, Ciudad de México, México; LACM: Natural History Museum, Los Angeles County Museum, Los Angeles, United
States of America (USA); and, MCZ: Museum of Comparative Zoology, Harvard University, Cambridge, USA.

When available, the geographical coordinates of the provided localities in the literature were validated using ArcMap 10.4.1 software, to corroborate distribution within the CMP maritime zone. Records were assigned to any of the following areas: coastlines of the states of Colima (COL), Jalisco (JAL), Michoacán (MICH), and Nayarit (NAY), and the islands Isabel (ISA), Marías (MAR), Marietas (MAT), and Revillagigedo (REV).

With this information, a checklist of the valid names and synonyms of Echinodermata from the CMP was constructed. The list includes those synonyms used exclusively in this area, yet some species may have larger synonym lists for other geographic areas. Systematics arrangements and valid names agree with Kroh and Mooi (2020), Mah (2020), Messing (2020), Stöhr, O'Hara, and Thuy (2020), and WoRMS (2020).

\section{RESULTS AND DISCUSSION}

\section{Historical review}

Crinoidea: Six references documented the Crinoidea from the CMP; one during the $19^{\text {th }}$, one in the $20^{\text {th }}$, and four in the $21^{\text {st }}$ century. Among the Echinodermata, Crinoidea represents the less studied class of the CMP. The study of the Crinoidea from the CMP began in 1895 when Hartlaub (1895) recorded the species Florometra tanneri (as Antedon rhomboidea) for MAR at $1236 \mathrm{~m}$ depth. Several years later, using a submersible, Roux (2004) collected one specimen of Hyocrinus foelli in $\mathrm{COL}$ at $3030 \mathrm{~m}$ depth. Finally, during deepwater dredging by the TALUD cruises aboard the R/V "El Puma" of the Universidad Nacional Autónoma de México, Florometra serratissima was collected in two stations located in COL between 1040 and $1106 \mathrm{~m}$ depth (Valdés de Anda et al., 2018). 
Asteroidea: 57 references addressed the Asteroidea distributed along the CMP. During the $19^{\text {th }}$ century only two works were published, meanwhile the $20^{\text {th }}$ and $21^{\text {st }}$ centuries presented similar numbers of works, 28 and 27 , respectively. The $19^{\text {th }}$ century represents the era with the lowest addition of Asteroidea. The first reported Asteroidea for the CMP were Luidia columbia and Astropecten regalis, both collected in San Blas, NAY (Gray, 1840).

The $20^{\text {th }}$ century was the period with the highest addition of Asteroidea to the CMP (25 species). Ludwig (1905) described Ampheraster marianus, Henricia gracilis, and Sarkaster validus (= Lophaster furcilliger) for MAR and other areas of the Eastern Pacific. According to samples from the Albatross, Fisher (1911) recorded Hippasteria lepidonotus and Henricia clarki for deep-waters from MAR and REV, respectively. Clark (1916) provided the original descriptions of Saraster insignis (= Nearchaster (Nearchaster) aciculosus) and Cyllaster seminuda (= Henricia seminudus) from Clarion Island, REV. In 1936, the Templeton Crocker Expedition collected echinoderms during the expedition of the Zaca along the north of México and as a result, Ziesenhenne (1937) mentioned Astropecten armatus, Acanthaster ellisii (= Acanthaster planci), Linckia columbiae, Pauliella aenigma (= Paulia horrida), and Sclerasterias heteropaes for shallow-waters (up to $100 \mathrm{~m}$ depth) from Clarion Island. During 1937-1938, the Zaca continued performing collections of shallow-water echinoderms, and Clark (1940) reported Luidia bellonae (Manzanillo, COL and Chamela Bay, JAL), Luidia foliolata, Pharia pyramidata, and Phataria unifascialis (Tenacatita Bay, JAL). Later, Caso (1943) reported the species Heliaster microbrachius and Luidia superba for Manzanillo, COL, as well as Nidorellia armata for the same locality and MAR. In later years, the addition of species for the CMP was sporadic, with mentions of just one species per work: Patiria miniata (Socorro Island, REV; Adem et al., 1960), Astropecten californicus (Manzanillo, COL; Caso, 1961), Mithrodia bradleyi (REV; Caso, 1962b), Pentaceraster cumingi (Socorro and Clarion Islands, REV; Hertlein, 1963), and Asteropsis carinifera (REV; Maluf, 1991).

During the $21^{\text {st }}$ century, only eight new records for the CMP have been documented. Fuentes-Farías et al. (2005) provided a list of echinoderms for $\mathrm{MICH}$ including the new records of Luidia tessellata and Heliaster helianthus. Two years later, the Mexican government decreed the MAR as a Biosphere Reserve, publishing a management and conservation program (CONANP, 2007a), which provided lists of the marine and terrestrial flora and fauna, and included the record of Echinaster (Othilia) tenuispina for the area. HoneyEscandón et al. (2008) and Solís-Marín et al. (2013b) provided the most-complete checklist of echinoderms for the Mexican Pacific and the Eastern Pacific, respectively, including the new records of Heliaster polybrachius (JAL), Mediaster transfuga and Meridiastra modesta (REV), and Narcissia gracilis (NAY). Recently, Nava-Bravo et al. (2019) included the record of Astropecten verrilli for MICH.

Ophiuroidea: 59 references have been published regarding this class. The $19^{\text {th }}$ century was the least productive since only one work was published. During the $20^{\text {th }}$ and $21^{\text {st }}$ centuries, similar numbers of manuscripts were published, 30 and 28, respectively. In 1891, the Albatross conducted an expedition along the Eastern Pacific, and as a result, Lütken and Mortensen (1899) described the first species and subspecies of ophiuroids from deep-waters of the CMP (MAR): Amphiura serpentina, Astrodia excavata, Dougaloplus notacanthus, Ophiacantha costata, Ophiacantha hirta, Ophiacantha moniliformis, Ophiernus adspersus annectens, Ophiomitra granifera, Ophiomitra partita, Ophiosphalma variabile, and Ophiura (Ophiura) scutellata.

During the $20^{\text {th }}$ century a total of 30 species were added to the list of ophiuroids of the CMP, representing the highest number, in comparison to the other two centuries accounted for in this review. Döderlein (1911) conducted a revision of the world's Euryalida, reporting Asteroschema sublaeve for MAR. 
With specimens collected in shallow-waters of Clarion Island, Ziesenhenne (1937) described Ophiacantha pyriformis, and reported for the first time in the CMP, the presence of Ophiactis savignyi, Ophiocoma aethiops, Ophioderma variegatum, Ophionereis annulata, and Ophiothrix galapagensis. Later, Clark (1940) found the conspicuous Diopederma daniana, Ophiolepis variegata, and Ophiothrix (Ophiothrix) spiculata in JAL and COL. In the same year, Ziesenhenne (1940) published the results obtained by the cruise Velero III performed in 1938, and described Amphichondrius laevis, Amphiodia sculptilis, Ophiolepis plateia, and Ophiophragmus lonchophorus, all of them collected in Tenacatita Bay, JAL. Later, the additions of new records of ophiuroids per work were reduced: Ophiocomella alexandri (REV; Steinbeck \& Ricketts, 1941), Ophioderma teres and Ophiolepis pacifica (Puerto Vallarta, JAL; Caso, 1951), Ophioderma panamense (REV; Ziesenhenne, 1955), Ophiomyxa panamensis (REV; Hertlein, 1963), Ophiostigma tenue (ISA; Clark, 1970), Ophiomusa lymani, Ophiothela mirabilis, and Ophiuroglypha irrorata irrorata (JAL and REV; Luke, 1982), Amphichondrius unamexici (= Amphichondrius granulatus; NAY; Caso, 1983a), Amphiura seminuda and Ophiophragmus marginatus (JAL and NAY; Caso, 1986b), Amphiura (Amphiura) assimilis and Gymnophiura mollis (MAR; Maluf, 1988), Ophiactis simplex (REV; Maluf, 1991), and Ophiuroconis bispinosa (REV; Hendler, 1996).

The addition of new records of ophiuroids (22) during the $21^{\text {st }}$ century has been important. Frontana-Uribe et al. (2000) collected specimens of Ophiocomella schmitti associated with coral rubble in localities from Socorro Island (REV). Fuentes-Farías et al. (2005) included the record of Ophiothrix (Ophiothrix) rudis for $\mathrm{MICH}$; in the same year, Maluf and Brusca (2005) published a checklist of the echinoderms from the Gulf of California, including the general distribution of the species, and the new records of Amphipholis elevata, Astrocaneum spinosum, Microphiopholis puntarenae, Ophiocomella sexradia, Ophiocnida hispida, Ophiolepis crassa, Ophiopholis bakeri, and
Ophiophthalmus diplasia (MAR and REV). The genus Ophiophthalmus is an invalid junior synonym and a new assignment is needed (Hendler, 1996), but in the present work we kept this temporary genus in order to avoid systematic confusions. Later, Honey-Escandón et al. (2008) reported 33 species of ophiuroids for the CMP, of which, the amphiurids Amphiodia occidentalis, Amphiodia platyspina, Amphiodia violacea, Amphiodia (Amphispina) digitata, Amphiodia (Amphispina) urtica, Amphipholis pugetana, and Microphiopholis platydisca, represented new records in several states and islands from the CMP. In 2011, Astrodictyum panamense (Ayala-Bocos, Reyes-Bonilla, Herrero-Pérezrul, Walther-Mendoza, \& CastañedaFernández de Lara, 2011) and Hemipholis gracilis (Hendler, 2011) were reported in REV and JAL, respectively. García-Hernández et al. (2014) compared the ecological diversity and species composition of benthic macroinvertebrates in rocky reefs of JAL and MAT, finding new records for Amphipholis squamata and Ophionereis perplexa. Finally, GranjaFernández et al. (2017) provided a checklist of ophiuroids from the CMP, adding the new record of Ophiophragmus papillatus.

Echinoidea: 73 references documented the presence of the Echinoidea from the CMP, representing the major number of works among all the classes. The $19^{\text {th }}$ century is represented only by two publications, the $20^{\text {th }}$ century for 39 , and the $21^{\text {st }}$ century for 32 works. In the $19^{\text {th }}$ century all the work regarding Echinoidea was published by Alexander Agassiz whom documented for the first time in the CMP the species Eucidaris thouarsii and Echinometra vanbrunti, both collected in Manzanillo, COL (Agassiz, 1872), and who described Brisaster townsendi and Plexechinus cinctus for stations located in deep-waters of MAR (Agassiz, 1898).

The $20^{\text {th }}$ century was by far the most productive in terms of new addition of echinoids (27) in the CMP. Clark described Hesperocidaris perplexa and Echinometra insularis (Clark, 1907; Clark, 1912), and reported Echinometra 
oblonga and Tripneustes gratilla from REV (Clark, 1912). With specimens collected by the Zaca, Ziesenhenne (1937) reported Clypeaster europacificus, Diadema mexicanum, and Tripneustes depressus in shallow-waters of Socorro and Clarion, REV. In 1938, was published an important work regarding fossil and living Echinoidea from the west American Cenozoic, in which were mentioned the new records of Astropyga pulvinata (JAL) and Toxopneustes roseus (ISA) (Grant \& Hertlein, 1938). Posteriorly, Clark (1940) added Clypeaster ochrus and Clypeaster speciosus from ISA and NAY, respectively, and Caso (1948a) included Arbacia stellata to NAY. Clark (1948) reported the highest number of new records of echinoids for the CMP (Brissopsis pacifica, Clypeaster rotundus, Encope micropora, Encope micropora insularis, Lovenia cordiformis, Meoma ventricosa grandis, and Rhyncholampas pacificus), based on the collections of the Velero III along the Eastern Pacific during the years 19311941; E. micropora insularis was described in this work and its holotype corresponds to Socorro Island, REV. The records in posterior works were sporadic: Hesperocidaris asteriscus (JAL and REV; Caso, 1961), Heterocentrotus mamillatus (REV; Chan, 1974), Dendraster excentricus and Lanthonia longifissa (ISA and COL, respectively; Caso, 1980), Agassizia scrobiculata (NAY; Caso, 1983b), and Mellita notabilis (MICH; Harold \& Telford, 1990).

Despite the number of published works during the $21^{\text {st }}$ century is similar to the previous century, only three new records for the area have been documented during the present era. These records correspond to species observed during field work and documented in the management and conservation programs of the National Park ISA (Centrostephanus coronatus; CONANP, 2005) and the Reserve of the Biosphere MAR (Brissus obesus; CONANP, 2007a). Finally, Ríos-Jara et al. (2008a) performed collections of echinoderms in ISA at different depths finding Brissus latecarinatus in shallow-waters $(<20 \mathrm{~m}$ depth).
Holothuroidea: 53 references mention holothuroids from the CMP. During the $19^{\text {th }}$ century only one work was published, 19 during the $20^{\text {th }}$ century, and 33 during the $21^{\text {st }}$ century. In the $19^{\text {th }}$ century only one work was published and therefore, the number of records was low. Despite, Laetmophasma fecundum (= Pannychia moseleyi), Psolus pauper, and Ypsilothuria bitentaculata were first mentioned in Ludwig (1893), it was until 1864 when Ludwig (1894) provided an extended description of the species, reporting them in deep waters from MAR; moreover, Molpadia musculus was recorded in this work too, for the same locality.

The $20^{\text {th }}$ century was the most productive in terms of the addition of new records (28). In 1926, the Templeton Crocker expedition aboard the Zaca performed dredges in order to obtain echinoderms along the Eastern Pacific, and collected Holothuria (Cystipus) inhabilis and Holothuria (Platyperona) difficilis in REV (Deichmann, 1937). From 1932 to 1938, the Allan Hancock Expedition aboard the Velero III collected several specimens of holothuroids and Deichmann (1941) found that Thyone bidentata and Thyone parafusus, both discovered in Tenacatita, JAL corresponded to new species, and that Leptopentacta panamica, Neothyone gibber, and Pentamera chierchiae were new records of species for the CMP (JAL and REV). Caso (1954) collected for the first time Holothuria (Selenkothuria) lubrica in Puerto Vallarta, JAL and found a similar species which was designated as a new species: Holothuria (Selenkothuria) portovallartensis. The biggest contribution of new records of holothuroids for the CMP (Holothuria (Halodeima) kefersteinii, Holothuria (Lessonothuria) pardalis, Holothuria (Mertensiothuria) hilla, Holothuria (Mertensiothuria) leucospilota, Holothuria (Semperothuria) imitans, Holothuria (Semperothuria) languens, Holothuria (Theelothuria) paraprinceps, Holothuria (Thymiosycia) arenicola, Holothuria (Thymiosycia) impatiens, and Isostichopus fuscus) was made by Deichmann (1958) which correspond to a continuation and expansion of the material collected by the Velero III and 
IV in JAL, as well as in ISA, MAR and REV. The new records per work during the rest of this century were reduced: Holothuria (Halodeima) inornata (Caso, 1961), Leptosynapta albicans (Caso, 1962b), Abyssocucumis abyssorum (Luke, 1982), Holothuria (Stauropora) fuscocinerea (Hickman, 1998), all of them collected in REV and NAY. Nepote-González (1998) conducted a research about the diversity of holothuroids of MAT, finding the new records of Chiridota rigida, Holothuria (Cystipus) rigida, Labidodemas americanum, and Pseudocnus californicus. Later, Solís-Marín and Laguarda-Figueras (1999) performed collections in several localities of the CMP (COL, NAY, MAT) and described a new species, Cucumaria flamma.

The addition of new records of holothuroids to the CMP has been important during the $21^{\text {st }}$ century. As part of the first program of management and conservation of REV National Park, CONANP (2004) documented Euapta godeffroyi. Fuentes-Farías et al. (2005) and HoneyEscandón et al. (2008) provided one of the most complete checklists of echinoderms in México and added the records of Neothyone gibbosa and Labidodemas maccullochi for $\mathrm{MICH}$, and Euthyonidiella zacae, Holothuria (Halodeima) atra, Holothuria (Mertensiothuria) viridiaurantia (= Holothuria (Mertensiothuria) hilla), Lissothuria ornata, Neocucumis veleronis, and Trachythyone peruana for several areas of the Mexican Pacific. Holothuria (Mertensiothuria) viridiaurantia is a recently described species which was previously assigned to $\mathrm{H}$. (Mertensiothuria) hilla (Borrero-Pérez \& VanegasGonzález, 2019). According to a preliminary revision of the material (unpublished data) and to Borrero-Pérez and Vanegas-González (2019), in the CMP, H. (Mertensiothuria) viridiaurantia inhabits in NAY, JAL, MICH and MAT. On the other hand, based on a taxonomical review of the material, we confirm that $H$. (Mertensiothuria) hilla inhabits in REV, but a taxonomic confirmation of the material from ISA and MAR is required (F.A. Solís-Marín, personal communication, 01 April 2020). Honey-Escandón et al. (2011) described Holothuria (Selenkothuria) carere, collected in shallow-waters of $\mathrm{MICH}$ and other states of the Mexican Pacific. Santos-Beltrán and Salazar-Silva (2011) provided images and an identification key of the holothuroids from Bahía de Banderas, NAY adding the record of Afrocucumis ovulum. Ríos-Jara et al. (2013) described the taxonomic composition of the echinoderms from shallow-waters in several localities of Bahía Chamela, JAL, adding Neopentamera anexigua, Pachythyone lugubris, and Pachythyone pseudolugubris to the CMP, and Solís-Marín, Arriaga-Ochoa, Galván-Villa, and Laguarda-Figueras (2018) described Lissothuria imbricata after the collection of material from the same area. In recent years, Nava-Bravo et al. (2019) elaborated the most complete checklist of echinoderms from $\mathrm{MICH}$ adding the records of Chiridota aponocrita, Epitomapta tabogae, and Thyonella mexicana.

Checklist: The present updated checklist includes 187 species of Echinodermata (three Crinoidea, 35 Asteroidea, 67 Ophiuroidea, 32 Echinoidea, and 50 Holothuroidea; Table 1) resulted from the revision of 137 literature references (3 445 records) and the taxonomic revision of Ophiuroidea deposited at museum collections. Additionally, we detected 57 records in the literature (two Crinoidea, 21 Asteroidea, 14 Ophiuroidea, 15 Echinoidea, and five Holothuroidea) considered as invalid and which were removed from the updated checklist. 
TABLE 1

Checklist of valid names (in bold) and synonyms of the Echinodermata from the Central Mexican Pacific based on literature review and museum records. All taxa are listed exactly as originally registered in the literature. Numbers refers to literature where taxa were listed, see References section. * = New distribution records

Phylum Echinodermata Bruguière, 1791

Class Crinoidea Miller, 1821

Order Comatulida A.H. Clark, 1908

Family Antedonidae Norman, 1865

Genus Florometra A.H. Clark, 1913

Florometra serratissima (A.H. Clark, 1907) COL 132

Florometra tanneri (Hartlaub, 1895) MAR 90, 92

Antedon rhomboidea Hartlaub, 189568

Orden Hyocrinida Rasmussen, 1978

Familia Hyocrinidae Carpenter, 1884

Genus Hyocrinus Thomson, 1876

Hyocrinus foelli Roux \& Pawson, 1999 COL 81, 114

Class Asteroidea de Blainville, 1830

Order Paxillosida Perrier, 1884

Family Luidiidae Sladen, 1889

Genus Luidia Forbes, 1839

Luidia bellonae Lütken, 1864 JAL, COL, MICH, REV 13, 39, 104

Luidia bellona Lütken, 1864 7,92

Luidia (Alternaster) bellona Lütken, $1864{ }^{91}$

Luidia columbia (Gray, 1840) NAY, COL, MICH, REV 13, 15, 92, 104, 126, 135

Luidia brevispina Lütken, $1871^{\mathbf{8}, 13}$

Luidia columbiae (Gray, 1840) 7, 117

Luidia (Petalaster) columbia (Gray, 1840) $\mathbf{5 8 , 8 1 , 9 1}$

Petalaster Columbia Gray, $1840{ }^{66}$

Luidia foliolata Grube, 1866 JAL $^{39}$

Luidia superba A.H. Clark, 1917 COL, MICH 8, 10, 13, 30, 124

Luidia (Alternaster) superba A.H. Clark, 1917 58, 81,91

Luidia tessellata Lütken, 1859 NAY, MICH, ISA 104

Luidia (Petalaster) tessellata Lütken, 1859 58, 81, 109, 110

Family Astropectinidae Gray, 1840

Genus Astropecten Gray, 1840

Astropecten armatus Gray, 1840 NAY, COL, MICH, REV 7, 8, 13, 15, 28, 30, 39 58, 81, 92, 104, 124, 135

Astropecten regalis Gray, 1840 NAY, COL, MICH 8, 13, 58, 66, 81, 104, 117, 124

Astropecten californicus Fisher, 1906 NAY, COL ${ }^{13,81,124}$

Astropecten verrilli de Loriol, 1899 MICH 104

Genus Psilaster Sladen, 1885

Psilaster armatus Ludwig, 1905 MAR ${ }^{86,90,92}$

Order Valvatida Perrier, 1884

Family Acanthasteridae Sladen, 1889

Genus Acanthaster Gervais, 1841

Acanthaster planci (Linnaeus, 1758) NAY, COL, MAR, ISA, MAT, REV 7, 32, 33, 41, 44, 45, 81, 107, 108, 120, 124 Acantaster planci (Linnaeus, 1758) ${ }^{126}$ 
Acantaster plansii (Linnaeus, 1758) ${ }^{31}$

Acanthaster elissi (Gray, 1840) ${ }^{\mathbf{1 3 4}}$

Acanthaster elisii pseudoplanci Caso, 196279

Acanthaster ellisi (Gray, 1840) $\mathbf{4 3 , 4 5}$

Acanthaster ellisii (Gray, 1840) 14, 15, 19, 41, 42, 72, 81, 92, 109, 110, 124, 135

Acanthaster ellisii pseudoplanci Caso, 1962 14, 15, 41, 69

Acanthaster pseudoplanci Caso, 196219

Family Asterinidae Gray, 1840

Genus Meridiastra O’Loughlin, 2002

Meridiastra modesta (Verrill, 1867) REV 81, 124

Asterina agustincasoi Caso, 197745

Genus Patiria Gray, 1840

Patiria miniata (Brandt, 1835) REV 120

Asterina miniata (Brandt, 1835) 1, 7, 15, 41, 45, 81

Patiria minata (Brandt, 1835) 126

Family Asterodiscididae Rowe, 1977

Genus Paulia Gray, 1840

Paulia horrida Gray, 1840 REV 41, 45, 91, 92, 126

Pauliella aenigma Ludwig, $1905^{\text {7, 13, 15, 77, } 135}$

Family Asteropseidae Hotchkiss \& A.M. Clark, 1976

Genus Asteropsis Müller \& Troschel, 1840

Asteropsis carinifera (Lamarck, 1816) JAL, ISA, REV 41, 45, 91, 92, 126

Asteropsis spinosa (Gray, 1840) ${ }^{\mathbf{8 1}}$

Gymnasteria spinosa Gray, 1840 109, 110

Family Goniasteridae Forbes, 1841

Genus Hippasteria Gray, 1840

Hippasteria lepidonotus (Fisher, 1905) MAR

Cryptopeltaster lepidonotus Fisher, 1905 13, 54, 90, 92

Hippasteria pacifica Ludwig, $1905^{\mathbf{5 4}}$

Genus Mediaster Stimpson, 1857

Mediaster transfuga Ludwig, 1905 REV 45, 120

Family Ophidiasteridae Verrill, 1870

Genus Linckia Nardo, 1834

Linckia columbiae Gray, 1840 NAY, JAL, MAR, REV 1, 7, 8, 13, 15, 41, 43, 45, 81, 91, 92, 126, 135

Genus Narcissia Gray, 1840

Narcissia gracilis A.H. Clark, 1916 NAY ${ }^{81}$

Genus Pharia Gray, 1840

Pharia pyramidata (Gray, 1840) NAY, JAL, COL, MICH, MAR, ISA, MAT, REV 7, 8, 13, 15, 39, 44, 73, 91, 92, 96, 104, 107, 113, 120, 129

Pharia piramidata (Gray, 1840) $\mathbf{4 2}$

Pharia pyramidatus (Gray, 1840) 33, 43, 45, 58, 72, 109, 110, 111, 112, 121, 124, 126

Pharia pyramidatus pyramidatus (Gray, 1840) ${ }^{\mathbf{8 1}}$

Pharia pyrimidata (Gray, 1840) ${ }^{87}$

Genus Phataria Gray, 1840

Phataria unifascialis (Gray, 1840) NAY, JAL, COL, MICH, MAR, ISA, MAT, REV 7, 15, 22, 29, 33, 39, 42, 43, 44, 45, 58, 72, $73,81,84,87,91,92,96,104,107,109,110,111,112,113,120,121,124,126,129$

Phataria unifacialis (Gray, 1840) ${ }^{8,13}$ 
Family Mithrodiidae Viguier, 1878

Genus Mithrodia Gray, 1840

Mithrodia bradleyi Verrill, 1867 NAY, COL, MICH, MAR, ISA, MAT, REV 7, 15, 33, 41, 43, 44, 45, 58, 79, 81, 87, 91, 92, 104, 107, 108, 109, 110, 120, 124, 126, 129, 134

Family Oreasteridae Fisher, 1908

Genus Nidorellia Gray, 1840

Nidorellia armata (Gray, 1840) JAL, COL, MICH, MAR, ISA, MAT, REV 58, 81, 104, 107, 108, 109, 110, 129

Nidoriella armata (Gray, 1840) 7,8,13

Genus Pentaceraster Döderlein, 1916

Pentaceraster cumingi (Gray, 1840) NAY, JAL, COL, MICH, ISA, MAT, REV 58, 81, 91, 92, 104, 107, 109, 110, 111, 113, 124, 126

Oreaster occidentalis Verrill, $1867^{7,77}$

Family Solasteridae Viguier, 1878

Genus Lophaster Verrill, 1878

Lophaster furcilliger Fisher, 1905 MAR

Lophaster validus (Ludwig, 1905) ${ }^{\mathbf{9 0}, 91}$

Sarkaster validus Ludwig, $1905^{\mathbf{8 6}}$

Order Forcipulatida Perrier, 1884

Family Asteriidae Gray, 1840

Genus Sclerasterias Perrier, 1891

Sclerasterias heteropaes Fisher, 1924 REV 7, 13, 15, 90, 92, 126, 135

Family Heliasteridae Viguier, 1879

Genus Heliaster Gray, 1840

Heliaster helianthus (Lamarck, 1816) NAY, MICH 58, 81, 104

Heliaster microbrachius Xantus, 1860 JAL, COL ${ }^{8,13,81}$

Heliaster polybrachius H.L. Clark, 1907 JAL $^{81}$

Family Pedicellasteridae Perrier, 1884

Genus Ampheraster Fisher, 1923

Ampheraster marianus (Ludwig, 1905) MAR 13, 55, 56, 90

Sporasterias mariana Ludwig, $1905^{86}$

Order Spinulosida Perrier, 1884

Family Echinasteridae Verrill, 1867

Genus Echinaster Müller \& Troschel, 1840

Subgenus Othilia Gray, 1840

Echinaster (Othilia) tenuispina Verrill, 1871 MAR

Echinaster tenuispina Verrill, $1871^{43}$

Echinaster (Othilia) teniuspinus Verrill, $1871^{\mathbf{8 1}}$

Genus Henricia Gray, 1840

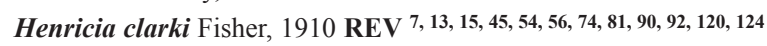

Henricia gracilis (Ludwig, 1905) MAR 90,91, 92

Cribrella gracilis Ludwig, $1905^{\mathbf{8 6}}$

Henricia seminudus (A.H. Clark, 1916) REV 45, 81, 120, 124

Cyllaster seminuda A.H. Clark, $1916^{\mathbf{1 3}, 15,34}$

Henricia seminuda (A.H. Clark, 1916) ${ }^{90}$

Order Notomyotida Ludwig, 1910

Family Benthopectinidae Verrill, 1899

Genus Nearchaster Fisher, 1911 
Subgenus Nearchaster Fisher, 1911

Nearchaster (Nearchaster) aciculosus (Fisher, 1910) REV

Nearchaster aciculosus (Fisher, 1910) 7,90,120

Saraster insignis A.H. Clark, $1916^{13,15,34}$

Class Ophiuroidea Gray, 1840

Order Euryalida Lamarck, 1816

Family Asteronychidae Ljungman, 1867

Genus Astrodia Verrill, 1899

Astrodia excavata (Lütken \& Mortensen, 1899) MAR 64, 92, 106

Asteronyx excavata Lütken \& Mortensen, 1899 13, 38, 50, 52, 63, 81, 88

Family Euryalidae Gray, 1840

Genus Asteroschema Örsted \& Lütken in Lütken, 1856

Asteroschema sublaeve Lütken \& Mortensen, 1899 MAR 50, 63, 64

Family Gorgonocephalidae Ljungman, 1867

Genus Astrocaneum Döderlein, 1911

Astrocaneum spinosum (Lyman, 1875) MAR 63, 64

Asterocaneum spinosum (Lyman, 1875) ${ }^{92}$

Genus Astrodictyum Döderlein, 1927

Astrodictyum panamense (Verrill, 1867) REV 6, 45, 63, 76

Order Ophiurida Müller \& Troschel, 1840, restricted sensu O’Hara, Hugall, Thuy, Stöhr, \& Martynov, 2017

Family Ophiosphalmidae O’Hara, Stöhr, Hugall, Thuy, \& Martynov, 2018

Genus Ophiosphalma H.L. Clark, 1941

Ophiosphalma variabile (Lütken \& Mortensen, 1899) JAL, COL, MAR, REV

Ophiomusium variabile Lütken \& Mortensen, 1899 45, 63, 64, 81, 88, 92, 120, 123, 124

Family Ophiomusaidae O'Hara, Stöhr, Hugall, Thuy, \& Martynov, 2018

Genus Ophiomusa Hertz, 1927

Ophiomusa lymani (Wyville-Thomson, 1873) MAR

Ophiomusium lymani Wyville-Thomson, 1873 63, 64, 87

Family Ophiuridae Müller \& Troschel, 1840

Genus Ophiura Lamarck, 1801

Subgenus Ophiura Lamarck, 1801

Ophiura (Ophiura) scutellata (Lütken \& Mortensen, 1899) MAR 63, 64

Homalophiura scutellata (Lütken \& Mortensen, 1899) ${ }^{\mathbf{3 8}, \mathbf{5 2}}$

Ophioglypha scutellata Lütken \& Mortensen, 189988

Ophiura scutellata (Lütken \& Mortensen, 1899) ${ }^{\mathbf{9 0 ,} 92}$

Genus Ophiuroglypha Hertz, 1927

Ophiuroglypha irrorata irrorata (Lyman, 1878) MAR

Ophiura (Ophiuroglypha) irrorata irrorata (Lyman, 1878) ${ }^{63,64}$

Ophiura irrorata (Lyman, 1878) ${ }^{\mathbf{8 7}}$

Family Ophiopyrgidae Perrier, 1893

Genus Gymnophiura Lütken \& Mortensen, 1899

Gymnophiura mollis Lütken \& Mortensen, 1899 MAR 63, 64, 90, 91, 92

Order Ophioscolecida O’Hara, Hugall, Thuy, Stöhr, \& Martynov, 2017

Family Ophioscolecidae Lütken, 1869 
Genus Ophiuroconis Matsumoto, 1915

Ophiuroconis bispinosa Ziesenhenne, 1937 JAL, COL, REV 45, 63, 64, 70, 81, 92, 120, 123, 124

Order Ophiacanthida O’Hara, Hugall, Thuy, Stöhr, \& Martynov, 2017

Family Ophiotomidae Paterson, 1985

Genus Ophiomitra Lyman, 1869

Ophiomitra granifera Lütken \& Mortensen, 1899 NAY, MAR ${ }^{63,64, ~ 87, ~} 88$

Ophiacantha granifera (Lütken \& Mortensen, 1899) 13

Ophioplinthaca granifera (Lütken \& Mortensen, 1899) 38, 52, 92

Ophiomitra partita Lütken \& Mortensen, 1899 MAR 63, 64, 88

Ophiacantha partita (Lütken \& Mortensen, 1899) ${ }^{13}$

Ophioplinthaca partita (Lütken \& Mortensen, 1899) ${ }^{38,52,92}$

Family Ophiacanthidae Ljungman, 1867

Genus Ophiacantha Müller \& Troschel, 1842

Ophiacantha costata Lütken \& Mortensen, 1899 MAR 13, 38, 52, 63, 64, 83, 88, 92

Ophiacantha hirta Lütken \& Mortensen, 1899 MAR 52, 63, 64, 81, 88, 92

Ophiacantha moniliformis Lütken \& Mortensen, 1899 MAR, REV 38, 45, 52, 63, 64, 81 88, 92, 120, 123, 124

Ophiacantha pyriformis Ziesenhenne, 1937 REV 7, 13, 15, 45, 52, 63, 90, 135

Genus Ophiophthalmus Matsumoto, 1917 (temporary genus)

Ophiophthalmus diplasia (H.L. Clark, 1911) REV

Ophiacantha diplasia H.L. Clark, 1911 45, 63, 92

Family Ophiodermatidae Ljungman, 1867

Genus Ophioderma Müller \& Troschel, 1840

Ophioderma panamense Lütken, 1859 NAY, JAL, COL, MICH, MAR, ISA, MAT, REV 7, 41, 42, 77, 79, 81, 84, 91, 92, 104, $109,110,111,123,124,137$

Ophiocryptus granulosus Nielsen, $1932^{81}$

Ophioderma panamensis Lütken, 1859 45, 62, 63, 64

Ophioderma teres (Lyman, 1860) NAY, JAL, COL, MAR, REV* 11, 13, 62, 63, 64, 81, 92

Ophioderma variegatum Lütken, 1856 NAY, JAL, COL, MAR, ISA*, REV 7, 11, 13, 15, 27, 29, 39, 41, 45, 77, 79, 81, 84, 91, 92, 111, 120, 124, 134, 135

Ophioderma variegata Lütken, $1856^{63,64,123,137}$

Genus Diopederma H.L. Clark, 1913

Diopederma daniana (Verrill, 1867) JAL, MICH ${ }^{63,64,104}$

Diopederma danianum (Verrill, 1867) ${ }^{\mathbf{1 1}, \mathbf{3 9}, \mathbf{5 8}, \mathbf{8 1}}$

Family Ophiomyxidae Ljungman, 1867

Genus Ophiomyxa Müller \& Troschel, 1842

Ophiomyxa panamensis Lütken \& Mortensen, $1899 \mathbf{R E V}^{\text {7, 63, 77, 91, } 92}$

Family Ophiocomidae Ljungman, 1867

Genus Ophiocoma L. Agassiz, 1836

Ophiocoma aethiops Lütken, 1859 NAY, JAL, COL, MICH, MAR, ISA, MAT, REV 7, 11, 13, 15, 29, 41, 42, 43, 44, 45, 58, 59, $62,63,64,79,81,84,87,91,92,104,109,110,111,120,121,122,123,124,130,134,135$

Genus Ophiocomella A.H. Clark, 1939

Ophiocomella alexandri (Lyman, 1860) NAY, JAL, COL, MICH, MAR, ISA, MAT, REV

Ophiocoma alexandri Lyman, 1860 7, 11, 13, 15, 29, 41, 42, 43, 44, 45, 49, 58, 59, 62, 63, 64, 79, 81, 84, 87, 91, 92, 104, 109, 110, 111, 120, 121, $123,124,130,134$

Ophiocomella schmitti A.H. Clark, 1939 NAY, COL, MAR*, REV 45, 57, 63, 64, 81, 123, 124

Ophiocomella sexradia (Duncan, 1887) MAR, REV 45, 63, 64, 92

Order Ophioleucida O’Hara, Hugall, Thuy, Stöhr, \& Martynov, 2017

Family Ophiernidae O’Hara, Stöhr, Hugall, Thuy, \& Martynov, 2018 
Genus Ophiernus Lyman, 1878

Ophiernus adspersus annectens Lütken \& Mortensen, 1899 MAR ${ }^{63,64,89}$

Ophiernus adspersus adspersus Lyman, $1883^{81}$

Ophiernus polyporum Lütken \& Mortensen, 1899 38, 52

Ophiernus polyporus Lütken \& Mortensen, 189988

Order Amphilepidida O’Hara, Hugall, Thuy, Stöhr, \& Martynov, 2017

Family Ophiolepididae Ljungman, 1867

Genus Ophiolepis Müller \& Troschel, 1840

Ophiolepis crassa Nielsen, 1932 COL*, MAR, ISA*, MAT 63, 64, 92

Ophiolepis pacifica Lütken, 1856 NAY, JAL, MICH*, MAR, MAT, REV* 62, 63, 64, 81

Ophiozona pacifica (Lütken, 1856) 11, 13, 29, 92

Ophiolepis plateia Ziesenhenne, 1940 JAL, ISA* 13, 52, 63, 64, 81, 115, 136

Ophiolepis variegata Lütken, 1856 NAY, JAL, COL, MAR 11, 39, 63, 64, 81, 87, 92, 123, 124

Family Ophionereididae Ljungman, 1867

Genus Ophionereis Lütken, 1859

Ophionereis annulata (Le Conte, 1851) NAY, JAL, COL, MICH, MAR, ISA, MAT, REV 7, 13, 15, 45, 58, 59, 61, 62, 63, 64, 81, 91, 92, 104, 109, 110, 111, 120, 123, 124, 130, 135

Ophiocoma annulata (Le Conte, 1851) ${ }^{43}$

Ophionereis dictiota Ziesenhenne, $1940^{41}$

Ophionereis dictyota Ziesenhenne, 1940 11, 45, 104, 123, 124, 134

Ophionereis perplexa Ziesenhenne, 1940 JAL, MAT 59

Family Amphiuridae Ljungman, 1867

Genus Amphiura Forbes, 1843

Amphiura seminuda Lütken \& Mortensen, 1899 NAY, REV 28, 63, 92

Amphiura serpentina Lütken \& Mortensen, 1899 MAR 52, 63, 64, 92, 131

Amphiura serpentina var. a Lütken \& Mortensen, $1899 \mathbf{8 8}$

Subgenus Amphiura (Amphiura) Forbes, 1843

Amphiura (Amphiura) assimilis Lütken \& Mortensen, 1899 MAR

Amphiodia assimilis (Lütken \& Mortensen, 1899) $\mathbf{6 3}^{\mathbf{6 3}} \mathbf{6 4 , 9 0}$

Amphiura assimilis Lütken \& Mortensen, $18999^{92}$

Genus Amphichondrius Nielsen, 1932

Amphichondrius granulatus (Lütken \& Mortensen, 1899) NAY

Amphichondrius unamexici Caso, 1983 25, 28, 90, 92

Amphichondrius laevis Ziesenhenne, 1940 JAL 52, 63, 64, 81, 87, 115, 136

Genus Amphiodia Verrill, 1899

Amphiodia occidentalis (Lyman, 1860) NAY 63, 64, 81

Amphiodia platyspina Nielsen, 1932 NAY 63, 64, 81

Amphiodia psara H.L. Clark, 1935 NAY*

Amphiodia sculptilis Ziesenhenne, 1940 JAL 52, 63, 64, 81, 136

Amphiodia tabogae Nielsen, 1932 NAY*

Amphiodia violacea (Lütken, 1856) NAY 63, 64, 81

Subgenus Amphispina Nielsen, 1932

Amphiodia (Amphispina) digitata Nielsen, 1932 NAY 63, 64, 81

Amphiodia (Amphispina) urtica (Lyman, 1860) NAY, MAR 63, 64, 81

Genus Amphipholis Ljungman, 1866

Amphipholis elevata Nielsen, 1932 MAR 63, 64, 92

Amphipholis pugetana (Lyman, 1860) NAY, REV* 63, 64, 81

Amphipholis squamata (Delle Chiaje, 1828) NAY, JAL, COL, MAT, ISA* 59, 63, 64 
Genus Dougaloplus A.M. Clark, 1970

Dougaloplus notacanthus (Lütken \& Mortensen, 1899) MAR ${ }^{63,64,92}$

Amphiura notacantha Lütken \& Mortensen, 1899 52, 81, 88

Genus Microphiopholis Turner, 1985

Microphiopholis geminata (Le Conte, 1851) NAY*

Microphiopholis platydisca (Nielsen, 1932) NAY, COL 63, 64

Amphipholis platydisca Nielsen, $1932{ }^{81}$

Microphiopholis puntarenae (Lütken, 1856) MAR ${ }^{63,64}$

Amphipholis puntarenae (Lütken, 1856) ${ }^{92}$

Genus Ophiocnida Lyman, 1865

Ophiocnida hispida (Le Conte, 1851) NAY*, JAL, COL, MAR, MAT 59, 62, 63, 64, 92

Genus Ophiophragmus Lyman, 1865

Ophiophragmus lonchophorus Ziesenhenne, 1940 NAY, JAL 13, 52, 63, 64, 90, 136

Ophiophragmus marginatus (Lütken, 1856) NAY, JAL 28, 63, 64, 81, 111

Ophiophragmus papillatus Ziesenhenne, 1940 NAY, JAL, MICH, MAT, REV* 64, 104

Ophiophragmus paucispinus Nielsen, 1932 MAR*

Genus Ophiostigma Lütken, 1856

Ophiostigma tenue Lütken, 1856 JAL, MAR, ISA, MAT 35, 63, 64, 92

Family Ophiactidae Matsumoto, 1915

Genus Ophiactis Lütken, 1856

Ophiactis savignyi (Müller \& Troschel, 1842) NAY, JAL, COL, MICH, MAR, ISA, MAT, REV 7, 11, 13, 23, 29, 45, 58, 59, 62, 63, 64, 81, 91, 104, 111, 120, 121, 122, 123, 124, 130, 135

Ophiactis simplex (Le Conte, 1851) NAY, JAL, COL, MICH, MAR, ISA, MAT, REV 58, 59, 62, 63, 64, 81, 91, 92, 104, 111

Genus Hemipholis Lyman, 1865

Hemipholis gracilis Verrill, 1867 NAY, JAL ${ }^{63,64,71}$

Family Ophiopholidae O‘Hara, Stöhr, Hugall, Thuy, \& Martynov, 2018

Genus Ophiopholis Müller \& Troschel, 1842

Ophiopholis bakeri McClendon, 1909 REV 63, 92

Family Ophiotrichidae Ljungman, 1867

Genus Ophiothrix Müller \& Troschel, 1840

Ophiothrix galapagensis Lütken \& Mortensen, 1899 MAR, REV 7, 13, 15, 45, 63, 64, 91, 92, 135

Subgenus Ophiothrix A.M. Clark, 1966

Ophiothrix (Ophiothrix) rudis Lyman, 1874 NAY, JAL, MICH, MAR, MAT, REV 62, 63, 64

Ophiothrix rudis Lyman, $1874 \mathbf{5 8 , 8 1 , 9 2 , 1 0 4}$

Ophiotrix rudis Lyman, $1874^{44}$

Ophiothrix (Ophiothrix) spiculata Le Conte, 1851 NAY, JAL, COL, MICH, MAR, ISA, MAT, REV 62, 63, 64, 104 Ophiothrix spiculata Le Conte, 1851 11, 13, 29, 39, 58, 59, 81, 87, 91, 92, 111, 123, 124

Genus Ophiothela Verrill, 1867

Ophiothela mirabilis Verrill, 1867 NAY, JAL, COL, MICH, ISA, MAT 58, 59, 62, 63, 64, 81, 104, 109, 110, 111

Ophiothrix mirabilis (Verrill, 1867) ${ }^{87}$

Class Echinoidea Leske, 1778

Order Cidaroida Claus, 1880

Family Cidaridae Gray, 1825

Genus Eucidaris Pomel, 1883

Eucidaris thouarsii (L. Agassiz \& Desor, 1846) NAY, JAL, COL, MICH, MAR, ISA, MAT, REV 7, 9, 13, 15, 21, 32, 33, $39,41,42,43,44,45,59,65,72,73,75,77,81,84,87,92,104,107,108,109,110,111,112,113,120,121,124,126,129,134,135$

Cidaris Thouarsii L. Agassiz \& Desor, $1846^{2}$

Eucidaris Thouarsii (L. Agassiz \& Desor, 1846) ${ }^{40}$ 
Eucidaris thourarsii (L. Agassiz \& Desor, 1846) ${ }^{91}$

Eucidaris thowarsii (L. Agassiz \& Desor, 1846) ${ }^{79}$

Genus Hesperocidaris Mortensen, 1928

Hesperocidaris asteriscus H.L. Clark, 1948 JAL, MICH, ISA, REV 7, 13, 15, 21, 45, 58, 92, 104, 111, 113, 126

Hesperocidaris perplexa (H.L. Clark, 1907) REV 7, 13, 15, 21, 45, 65, 81, 92, 97, 120, 124, 135

Tretocidaris perplexa H.L. Clark, $1907^{36,51}$

Order Diadematoida Duncan, 1889

Family Diadematidae Gray, 1855

Genus Astropyga Gray, 1825

Astropyga pulvinata (Lamarck, 1816) JAL, COL, MICH, MAR, MAT, REV 9, 21, 33, 41, 44, 45, 58, 65, 73, 81, 87, 91, 92, 104, 107,113

Astropygia pulvinata (Lamarck, 1816) ${ }^{\mathbf{1 2 6}}$

Genus Centrostephanus Peters, 1855

Centrostephanus coronatus (Verrill, 1867) JAL, COL, MICH, MAR, ISA, MAT 42, 43, 44, 72, 73, 81, 96, 107, 109, 110, 111, 112, $113,124,129$

Genus Diadema Gray, 1825

Diadema mexicanum A. Agassiz, 1863 NAY, JAL, COL, MICH, MAR, ISA, MAT, REV 1, 5, 7, 13, 15, 21, 22, 29, 31, 33, 41, $42,43,44,45,53,58,72,73,74,75,79,81,84,91,92,96,98,104,107,108,109,110,111,112,113,120,121,124,126,129,134$

Centrechinus mexicanus (A. Agassiz, 1863) 7,9, 40, 65, 77, 135

Diadema mexicanus A. Agassiz, $1863^{31}$

Order Arbacioida Gregory, 1900

Family Arbaciidae Gray, 1855

Genus Arbacia Gray, 1835

Arbacia stellata (Blainville, 1825; ?Gmelin, 1788) NAY, JAL, MAR, ISA

Arbacia incisa (A. Agassiz, 1863) 9, 13, 21, 42, 43, 81, 84, 109, 110, 111, 113

Order Camarodonta Jackson, 1912

Family Echinometridae Gray, 1855

Genus Echinometra Gray, 1825

Echinometra insularis H.L. Clark, 1912 REV $^{37,51,100}$

Echinometra oblonga (Blainville, 1825) REV 1, 7, 13, 15, 21, 31, 37, 40, 41, 45, 74, 79, 81, 91, 92, 95, 120, 124, 126, 134, 135 Echinometra mathei oblonga (Blainville, 1825) ${ }^{75}$

Echinometra vanbrunti A. Agassiz, 1863 NAY, JAL, COL, MICH, MAR, ISA, MAT, REV 1, 7, 15, 31, 33, 41, 42, 43, 44, $45,58,73,79,81,84,87,91,92,104,107,108,109,110,111,113,120,121,124,126,129,134$

Echinometra van brunti A. Agassiz, 1863 13, 21, 22, 29

Echinometra Van brunti A. Agassiz, 1863 2,9

Echinometra van-brunti A. Agassiz, 186377

Echinometra VanBrunti A. Agassiz, $1863{ }^{40}$

Echinometra vanbruntii A. Agassiz, $1863^{\mathbf{8 0}}$

Heliocidaris stenopora H.L. Clark, $1912^{9}$

Genus Heterocentrotus Brandt, 1835

Heterocentrotus mamillatus (Linnaeus, 1758) REV 7, 32, 108

Family Toxopneustidae Troschel, 1872

Genus Toxopneustes L. Agassiz, 1841

Toxopneustes roseus (A. Agassiz, 1863) NAY, JAL, COL, MICH, MAR, ISA, MAT, REV 7, 13, 15, 21, 22, 29, 33, 40, 41, 42, $43,44,45,58,65,73,79,81,87,91,92,96,104,107,109,110,111,112,113,121,124,126,129$ 
Genus Tripneustes L. Agassiz, 1841

Tripneustes depressus A. Agassiz, 1863 JAL, COL, MICH, MAR, ISA, MAT, REV 1, 7, 13, 15, 18, 20, 21, 31, 33, 40, 41, 42, $43,44,45,65,73,75,79,80,81,91,92,99,104,107,108,109,110,111,113,120,121,124,126,129,130,134,135$

Tripneustes gratilla (Linnaeus, 1758) REV ${ }^{37}$

Order Cassiduloida Claus, 1880

Family Cassidulidae L. Agassiz \& Desor, 1847

Genus Rhyncholampas A. Agassiz, 1869

Rhyncholampas pacificus (A. Agassiz, 1863) MAR, REV 93

Cassidulus pacifica (A. Agassiz, 1863) 7,91,92

Cassidulus pacificus (A. Agassiz, 1863) 13, 15, 26, 40, 101

Order Clypeasteroida A. Agassiz, 1872

Family Clypeasteridae L. Agassiz, 1835

Genus Clypeaster Lamarck, 1801

Clypeaster europacificus H.L. Clark, 1914 REV 7, 13, 15, 24, 40, 51, 81, 92, 120, 124, 135

Clypeaster (Alexandria) europacificus H.L. Clark, $1914{ }^{102}$

Clypeaster ochrus H.L. Clark, 1914 MAR, ISA, REV 13, 24, 39, 81, 92, 93, 120, 124, 126

Clypeaster (Stolonoclypus) ochrus H.L. Clark, $1914{ }^{91}$

Clypeaster rotundus (A. Agassiz, 1863) JAL, MAR, ISA, REV 7, 15, 24, 40, 43, 77, 92

Clypeaster (Stolonoclypus) rotundus A. Agassiz, 1863 91, 102

Clypeaster speciosus Verrill, 1870 NAY, COL, REV 7, 13, 15, 24, 39, 40, 41, 45, 77, 92, 126

Clypeaster (Stolonoclypus) speciosus Verrill, 1870 91, 102

Family Dendrasteridae Lambert, 1900

Genus Dendraster L. Agassiz in Agassiz \& Desor, 1847

Dendraster excentricus (Eschscholtz, 1831) ISA ${ }^{24}$

Family Mellitidae Stefanini, 1912

Genus Mellita L. Agassiz, 1841

Mellita notabilis H.L. Clark, 1947 MICH 58, 67, 81, 104

Genus Encope L. Agassiz, 1840

Encope micropora L. Agassiz, 1841 JAL, MICH

Encope fragilis H.L. Clark, 194840

Encope micropora fragilis H.L. Clark, $1948{ }^{24}$

Encope perspectiva L. Agassiz, 1841 40, 58, 81, 104

Encope wetmorei A.H. Clark, $1946^{\mathbf{5 8 , 8 1 , 1 0 4}}$

Encope micropora insularis H.L. Clark, 1948 REV 24, 102

Encope insularis H.L. Clark, 1948 13, 15, 40, 51

Genus Lanthonia Coppard, 2016

Lanthonia longifissa (Michelin, 1858) COL

Mellita longifissa Michelin, $1858^{24,81,124}$

Order Holasteroida Durham \& Melville, 1957

Family Plexechinidae Mooi \& David, 1996

Genus Plexechinus A. Agassiz, 1898

Plexechinus cinctus A. Agassiz, 1898 MAR $^{\text {3, 4, 13, 26, 51, 90, } 92}$

Order Spatangoida L. Agassiz, 1840

Family Schizasteridae Lambert, 1905

Genus Brisaster Gray, 1855 


\section{Brisaster townsendi (A. Agassiz, 1898) MAR}

Briaster townsendi (A. Agassiz, 1898) ${ }^{\mathbf{8 1}}$

Brisaster towsendi (A. Agassiz, 1898) 13, 26

Schizaster townsendi A. Agassiz, 189851

Schizaster Townsendi A. Agassiz, $1898^{3,4}$

Family Prenasteridae Lambert, 1905

Genus Agassizia Valenciennes, 1846

Agassizia scrobiculata Valenciennes, 1846 NAY 26, 81

Family Brissidae Gray, 1855

Genus Brissopsis L. Agassiz, 1840

Brissopsis pacifica (A. Agassiz, 1898) REV 7, 13, 15, 26, 40, 91, 92

Genus Brissus Gray, 1825

Brissus latecarinatus (Leske, 1778) ISA 109, 110

Brissus obesus Verrill, 1867 MAR ${ }^{43}$

Genus Meoma Gray, 1851

Meoma ventricosa grandis Gray, 1851 JAL, REV 81, 91, 93, 120

Meoma grandis Gray, 1851 7, 26, 40, 77, 92, 103

Meoma ventricosa (Lamarck, 1816) ${ }^{45,124,126}$

Family Loveniidae Lambert, 1905

Genus Lovenia Desor in Agassiz \& Desor, 1847

Lovenia cordiformis A. Agassiz, 1872 REV 13, 15, 26, 40, 41, 45, 77, 81, 92, 93, 124

Lovenia cardiformis A. Agassiz, $1872^{7}$

Lovenia (Lovenia) cordiformis A. Agassiz, $1872{ }^{91}$

Class Holothuroidea Selenka, 1867

Order Dendrochirotida Grube, 1840

Family Psolidae Burmeister, 1837

Genus Lissothuria Verrill, 1867

Lissothuria imbricata Solís-Marín, Arriaga-Ochoa, Galván-Villa, \& Laguarda-Figueras, 2018 JAL 125

Lissothuria ornata Verrill, 1867 JAL, MICH, MAT 104, 111, 113, 125, 127

Thyonepsolus beebei Deichmann, $1937^{\mathbf{8 1}}$

Genus Psolus Oken, 1815

Psolus pauper Ludwig, 1893 MAR ${ }^{85,94}$

Family Sclerodactylidae Panning, 1949

Genus Afrocucumis Deichmann, 1944

Afrocucumis ovulum (Selenka, 1867) NAY, JAL, MICH, MAT 104, 113, 116, 127

Genus Neothyone Deichmann, 1941

Neothyone gibber (Selenka, 1867) NAY, JAL, MICH, MAT, REV 7, 44, 47, 81, 84, 90, 104, 105, 111, 112, 113, 128 Thyone gibber (Selenka, 1867) 13, 15

Neothyone gibbosa Deichmann, 1941 JAL, MICH 58, 81, 104, 111, 113

Genus Pachythyone Deichmann, 1941

Pachythyone lugubris (Deichmann, 1939) JAL 111, 113

Pachythyone pseudolugubris Deichmann, 1941 JAL, MICH, MAT 104, 111, 113, 127

Genus Neopentamera Deichmann, 1941

Neopentamera anexigua Deichmann, 1941 JAL 111, 113

Genus Euthyonidiella Heding \& Panning, 1954

Euthyonidiella zacae (Deichmann, 1938) REV 120

Phyllophorus zacae Deichmann, 1938 45, 81, 124 
Family Phyllophoridae Östergren, 1907

Genus Pentamera Ayres, 1852

Pentamera chierchiae (Ludwig, 1886) JAL, MICH, MAT, REV

Pentamera chierchia (Ludwig, 1886) 7, 13, 15, 45, 47, 81, 91, 92, 104, 111, 113, 124, 126, 128

Genus Thyone Oken, 1815

Thyone bidentata Deichmann, 1941 JAL 13, 47, 113, 119

Thyone parafusus Deichmann, 1941 JAL 13, 47, 90, 92, 119, 130

Family Cucumariidae Ludwig, 1894

Genus Abyssocucumis Heding, 1942

Abyssocucumis abyssorum (Théel, 1886) NAY ${ }^{87}$

Genus Cucumaria de Blainville, 1830

Cucumaria flamma Solís-Marín \& Laguarda-Figueras, 1999 NAY, JAL, COL, MICH, MAT 44, 58, 73, 81, 104, 107, 111, 113, 118, 121, 124, 128, 129

Pattalus mollis Selenka, $1868^{44,105}$

Genus Neocucumis Deichmann, 1944

Neocucumis veleronis (Deichmann, 1941) JAL, COL, MICH, MAT 81, 104, 111, 113, 124, 127

Genus Pseudocnus Panning, 1949

Pseudocnus californicus (Semper, 1868) NAY, JAL, COL, MICH, MAT 44, 58, 81, 104, 105, 111, 113, 116, 124, 128

Genus Trachythyone Studer, 1876

Trachythyone peruana (Semper, 1868) NAY, JAL ${ }^{81,113}$

Genus Thyonella Verrill, 1872

Thyonella mexicana (Deichmann, 1941) MICH 104

Genus Leptopentacta H.L. Clark, 1938

Leptopentacta panamica Deichmann, 1941 JAL 13, 47, 90

Family Ypsilothuriidae Heding, 1942

Genus Ypsilothuria Perrier, 1886

Ypsilothuria bitentaculata (Ludwig, 1893) MAR 92, 94

Shpaerothuria bitentaculata Ludwig, $1893{ }^{85}$

Order Holothuriida Miller, Kerr, Paulay, Reich, Wilson, Carvajal \& Rouse, 2017

Family Holothuriidae Burmeister, 1837

Genus Holothuria Linnaeus, 1767

Subgenus Cystipus Haacke, 1880

Holothuria (Cystipus) inhabilis Selenka, 1867 REV 91, 126

Holothuria inhabilis Selenka, $1867^{\text {7, 46, 92, } 130}$

Holothuria parinhabilis Cherbonnier, $1951^{15}$

Jaegerothuria inhabilis (Selenka, 1867) ${ }^{77}$

Holothuria (Cystipus) rigida (Selenka, 1867) NAY, JAL, COL, MICH, MAT 44, 58, 81, 104, 105, 113, 124 Holothuria rigida (Selenka, 1867) ${ }^{44,84,111}$

Subgenus Halodeima Pearson, 1914

Holothuria (Halodeima) atra Jaeger, 1833 NAY, JAL, ISA ${ }^{81}$

Holothuria atra Jaeger, 1833 109, 110

Holothuria (Halodeima) inornata Semper, 1868 NAY, JAL, MICH, ISA, MAT, REV 104, 107, 113, 116, 119, 120, 126, 127 Holothuria inornata Semper, 1868 13, 15, 111, 112, 129

Holothuria (Halodeima) kefersteinii (Selenka, 1867) NAY, JAL, MICH, MAR, ISA, MAT, REV 104, 107 Holothuria kefersteiini (Selenka, 1867) ${ }^{43}$

Holothuria kefersteini (Selenka, 1867) 44, 45, 84, 92

Holothuria kefersteinii (Selenka, 1867) 129

Holothuria kefersteni (Selenka, 1867) ${ }^{7}$ 
Holothuria (Halodeima) kefersteini (Selenka, 1867) 58, 81, 91, 105, 109, 110, 116, 119, 120, 124, 126

Holothuria (Paraholothuria) riojai Caso, 196358

Ludwigothuria kefersteini (Selenka, 1867) ${ }^{48,77}$

Subgenus Lessonothuria Deichmann, 1958

Holothuria (Lessonothuria) pardalis Selenka, 1867 JAL 91, 113

Holothuria pardalis Selenka, $1867^{13,90}$

Lessonothuria pardalis (Selenka, 1867) ${ }^{48}$

Subgenus Mertensiothuria Deichmann, 1958

Holothuria (Mertensiothuria) hilla Lesson, 1830 MAR, ISA, REV 120, 126

Brandtothuria gyrifer (Selenka, 1867) ${ }^{48}$

Holothuria gyrifer (Selenka, 1867) ${ }^{\mathbf{1 3}, 16}$

Holothuria hilla Lesson, 1830 45, 111

Holothuria (Thymiosycia) hilla Lesson, 1830 81, 109, 110, 124

Holothuria (Mertensiothuria) leucospilota (Brandt, 1835) COL, MAR, REV 81, 91, 120, 124, 126 Holothuria leucospilota (Brandt, 1835) 7, 13, 15, 31, 41, 45, 79, 90, 92

Mertensiothuria leucospilota (Brandt, 1835) ${ }^{48}$

Holothuria (Mertensiothuria) viridiaurantia Borrero-Pérez \& Vanegas-González, 2019 NAY, JAL, MICH, MAT Holothuria hilla Lesson, 1830 111, 129

Holothuria (Mertensiothuria) hilla Lesson, 1830 104, 113, 116, 127

Holothuria (Thymiosycia) hilla Lesson, $1830^{\mathbf{8 1}}$

Subgenus Platyperona Rowe, 1969

Holothuria (Platyperona) difficilis Semper, 1868 NAY, JAL, MICH, MAR, MAT, REV 81, 91, 104, 116, 120, 124, 126, 127 Holothuria difficilis Semper, 1868 7, 13, 15, 45, 46, 112, 129, 130

Microthele difficilis (Semper, 1868) 48

Subgenus Selenkothuria Deichmann, 1958

Holothuria (Selenkothuria) carere Honey-Escandón, Solís-Marín, \& Laguarda-Figueras, 2011 MICH 82, 104

Holothuria (Selenkothuria) lubrica Selenka, 1867 NAY, JAL, MICH, MAR, ISA, REV 58, 81, 91, 104, 109, 110, 113, 116, 120, 124, 126

Holothuria lubrica Selenka, 1867 7, 12, 13, 15, 45, 58, 92, 111, 112, 121

Holothuria pseudolubrica Cherbonnier, $1951^{15}$

Selenkothuria lubrica (Selenka, 1867) ${ }^{\mathbf{4 1}, 48}$

Holothuria (Selenkothuria) portovallartensis Caso, 1954 NAY, JAL, MICH, REV 58, 81, 91, 104, 116, 119, 126 Holothuria portovallartensis Caso, 1954 12, 13, 92

Selenkothuria portovallartensis (Caso, 1954) 48

Subgenus Semperothuria Deichmann, 1958

Holothuria (Semperothuria) imitans Ludwig, 1875 NAY, JAL, COL, MICH, ISA, MAT, REV 81, 91, 104, 105, 109, 110, $116,120,124,126$

Holothuria imitans Ludwig, 1875 7, 13, 15, 44, 45, 92, 129

Holothuria imitans var. polymorpha Caso, 196215

Semperothuria imitans (Ludwig, 1875) 48, 77

Holothuria (Semperothuria) languens Selenka, 1867 JAL, MICH, MAR 81, 104

Holothuria languens Selenka, 186713

Semperothuria languens (Selenka, 1867) ${ }^{48}$

Subgenus Stauropora Rowe, 1969

Holothuria (Stauropora) fuscocinerea Jaeger, 1833 JAL, MICH, MAR, ISA, REV

Holothuria fuscocinerea Jaeger, 1833 45, 73, 78

Holothuria fuscoscinerea Jaeger, $1833^{43}$

Holothuria (Mertensiothuria) fuscocinerea Jaeger, 1833 81, 109, 110, 124

Holothuria (Stauropora) pluricuriosa Deichmann, 1937 120, 126 
Subgenus Theelothuria Deichmann, 1958

Holothuria (Theelothuria) paraprinceps Deichmann, 1937 REV ${ }^{126}$

Holothuria paraprinceps Deichmann, $1937{ }^{92}$

Theelothuria paraprinceps (Deichmann, 1937) 48, 77

Subgenus Thymiosycia Pearson, 1914

Holothuria (Thymiosycia) arenicola Semper, 1868 NAY, JAL, COL, MICH, MAR, ISA, MAT, REV 44, 58, 81, 91, 104, $105,107,109,110,113,116,119,120,124,126$

Brandtothuria arenicola (Semper, 1868) ${ }^{\mathbf{4 2}, 48,77}$

Holothuria arenicola Semper, 1868 7, 15, 44, 45, 73, 84, 92, 111, 121, 129

Holothuria (Thymiosycia) impatiens (Forskål, 1775) NAY, JAL, COL, MICH, MAR, ISA, MAT, REV 58, 81, 91, 104, $105,107,109,110,113,116,120,124$

Brandtothuria impatiens (Forskål, 1775) $\mathbf{4 2 , 4 8 , 7 7}$

Holothuria impatiens (Forskål, 1775) 7, 15, 43, 44, 45, 58, 84, 92, 111, 112, 124, 129

Genus Labidodemas Selenka, 1867

Labidodemas americanum Deichmann, 1938 NAY, JAL, COL, MICH, MAT, REV 44, 45, 81, 104, 105, 113, 116, 120, 124, 126, 129

Labidodemas americanus Deichmann, $1938{ }^{\mathbf{5 8}}$

Labidodemas maccullochi (Deichmann, 1958) JAL, COL, MICH 111, 113

Holothuria (Irenothuria) maccullochi (Deichmann, 1958) 58

Labidodemas macullochi (Deichmann, 1958) ${ }^{\mathbf{8 1}, 124}$

Order Synallactida Miller, Kerr, Paulay, Reich, Wilson, Carvajal, \& Rouse, 2017

Family Stichopodidae Haeckel, 1896

Genus Isostichopus Deichmann, 1958

Isostichopus fuscus (Ludwig, 1875) NAY, JAL, COL, MICH, MAR, ISA, MAT, REV 7, 42, 43, 44, 45, 48, 58, 72, 73, 81, 84, 91, 92, 104, 105, 107, 109, 110, 111, 112, 113, 119, 120, 121, 124, 126, 129, 133

Isostichopus fuscis (Ludwig, 1875) ${ }^{\mathbf{9 6}}$

Stichopus fuscus Ludwig, $1875^{15,17,78}$

Order Elasipodida Théel, 1882

Family Laetmogonidae Ekman, 1926

Genus Pannychia Théel, 1882

Pannychia moseleyi Théel, 1882 JAL, COL, MAR 92, 94

Laetmophasma fecundum Ludwig, 189385

Order Molpadida Haeckel, 1896

Family Molpadiidae Müller, 1850

Genus Molpadia Cuvier, 1817

Molpadia musculus Risso, 1826 MAR ${ }^{92}$

Ankyroderma danielsseni Théel, $1886^{\mathbf{8 5}}$

Order Apodida Brandt, 1835

Family Chiridotidae Östergren, 1898

Genus Chiridota Eschscholtz, 1829

Chiridota aponocrita A.H. Clark, 1920 MICH ${ }^{104}$

Chiridota rigida Semper, 1867 NAY, MICH, MAT 44, 81, 104, 105

Family Synaptidae Burmeister, 1837

Genus Epitomapta Heding, 1928

Epitomapta tabogae Heding, 1928 MICH ${ }^{104}$

Genus Euapta Östergren, 1898 


\author{
Euapta godeffroyi (Semper, 1868) NAY, JAL, COL, MICH, MAR, ISA, REV 41, 43, 45, 60, 73, 81, 109, 110, 113 \\ Genus Leptosynapta Verrill, 1867 \\ Leptosynapta albicans (Selenka, 1867) REV ${ }^{7,15,90,94}$
}

$\mathrm{COL}=$ Colima ISA = Isabel Island; JAL = Jalisco; MAR = Marías Islands; MAT = Marietas Islands; MICH = Michoacán; $\mathrm{NAY}=$ Nayarit; REV $=$ Revillagigedo Islands.

Invalid and doubtful records: The records considered as invalid (52) or doubtful (five) are based on: 1) erroneous or doubtful distributions, 2) incorrect taxonomic identifications, or 3) entries in faunal list not backed up by records in the primary literature.

There are some records considered invalid because of inconsistencies in their geographical distributions. The ophiuroids Ophiocomella ophiactoides and Astrophyton muricatum, and the holothuroid Isostichopus badionotus have been reported in the CMP (Honey-Escandón et al., 2008; Solís-Marín et al., 2016b; CONANP, 2019), yet the species distribution range is exclusively for the Caribbean and the Gulf of México (Hendler, Miller, Pawson, \& Kier, 1995; Solís-Marín et al., 2013a), meanwhile the echinoids Echinothrix calamaris and Echinothrix diadema inhabits the Indian Ocean, but have been reported in REV (Sotelo-Casas \& Rodríguez-Troncoso, 2014). The holothuroid Pentamera chiloensis is reported in Barra de Navidad, JAL (Rodríguez-Troncoso et al., 2013); this species distributes only in South America (Perú and Chile; Solís-Marín et al., 2013a). Records previously mentioned correspond to erroneous records and must be invalid (R.C. Sotelo-Casas and F.A. Solís-Marín, personal communication, 01 March 2020).

The crinoid Hyocrinus foelli (Solís-Marín et al., 2013a; Solís-Marín et al., 2013b; SolísMarín, Laguarda-Figueras, \& Honey-Escandón, 2014) and the holothuroid Psychronaetes hanseni (Maluf, 1991; Bautista-Romero et al., 1994; Massin \& Hendrickx, 2011) have been reported in Clarion Island, REV, but according to their original descriptions (Pawson, 1983; Roux \& Pawson, 1999) and the validation of the geographical coordinates, all the records corresponds to the Clarion-Clipperton Fracture Zone which is located approximately $1200 \mathrm{~km}$ far away from REV. The echinoids Aporocidaris milleri and Kamptosoma asterias were reported in NAY (station 96, 2988-3001 m depth) by Parker (1963), yet the validation of the geographic coordinates revealed that this station is located in the entrance of the Gulf of California, and far away from the maritime zone of NAY. Moreover, $K$. asterias is distributed in the Central Pacific Ocean, Chile and the Antarctica (Mooi, Constable, Lockhart, \& Pearse, 2004).

Furthermore, some records had inconsistencies in their taxonomic identifications. Ophionereis albomaculata (ophiuroid) has records in ISA and MAR (Caso, 1961; Maluf, 1991; Maluf \& Brusca, 2005; Granja-Fernández et al., 2015a; Granja-Fernández et al., 2017); during our revision of museum collections, we taxonomically reviewed all the material known for O. albomaculata (Galápagos Islands and Panamá), and none corresponded to México. The material identified as $O$. albomaculata from México must relate to another species of Ophionereis. The collected material of the holothuroid Holothuria (Lessonothuria) pardalis from REV (Solís-Marín et al., 2013a; Sotelo-Casas \& Rodríguez-Troncoso, 2014), was previously identified as $H$. (Lessenothuria) pardalis, yet it corresponds to a new species in process of description (F.A. Solís-Marín, personal communication, 01 April 2020). It is important to mention that in the CMP, the species only distributes in Tenacatita, JAL (Deichmann, 1958).

Most of the entries are based on unbacked up records of primary references. BautistaRomero et al. (1994) constructed a checklist of echinoderms from REV according to previous records (Fisher, 1911; Ziesenhenne, 1937; Caso, 1962b; Maluf, 1988), and reported: Florometra serratissima (crinoid); Amphiaster insignis, 
Astropecten verrilli, Echinaster (Othilia) tenuispina, Heliaster kubiniji, Henricia aspera, Henricia asthenactis, Henricia leviuscula, Henricia polyacantha, Leiaster teres, Luidia foliolata, Luidia phragma, Mediaster aequalis, Narcissia gracilis, Odontaster crassus, Tethyaster canaliculatus, and Thrissacanthias penicillatus (asteroids); Amphiodia (Amphispina) urtica, Amphipholis squamata, Amphiura arcystata, Astrocaneum spinosum, Diopederma daniana, Ophiolepis crassa, Ophionereis eurybrachiplax, Ophiopaepale diplax and Ophiura luetkenii (ophiuroids); and Arbacia stellata (as Arbacia incisa), Centrostephanus coronatus, Encope grandis, Encope micropora, Lytechinus pictus, and Strongylocentrotus fragilis (echinoids). We consulted the previous literature and we did not find these records on them or in other literature references, therefore these records are invalid.

Based on literature records, Lophaster furcilliger (asteroid), Astrocaneum spinosum (ophiuroid), and Centrostephanus coronatus (echinoid) have been recorded in the program of management and conservation of REV (CONANP, 2019). The only records of L. furcilliger in the CMP correspond to MAR (Ludwig, 1905; Maluf, 1988; Maluf, 1991), therefore their inclusion in REV is incorrect. Despite it is very probable that $A$. spinosum and $C$. coronatus distributes in REV, we did not find any record in publications to back it up. Other species reported in a program of management and conservation (ISA; CONANP, 2005) are the echinoids Clypeaster europacificus and Lovenia cordiformis, but again no primary references supporting these records were found, and therefore are considered as invalid records.

Finally, based on a revision of literature, the asteroids Paulia horrida and Luidia bellonae were reported in MAR by Maluf (1988) and Maluf and Brusca (2005), respectively; nevertheless, we did not find any record of these species for MAR but for REV. Similarly, the asteroid Cnemidaster nudus has only one mention for COL (Solís-Marín et al., 2016b), but it corresponds to an erroneous record (F.A. Solís-Marín, personal communication, 01 April
2020). For these reasons, we consider both records as invalid.

The following records were considered as doubtful. The ophiuroid Amphiodia periercta inhabits in the North Pacific Ocean (from Aleutian Islands, Alaska to central California; Lambert \& Austin, 2007), yet it has a report in Clarion Island, REV (Maluf, 1988; BautistaRomero et al., 1994). Due to the northern affinity of $A$. periercta, its distribution in REV is unlikely. The record of Hymenaster pentagonalis in REV was obtained by the Expedition Ocean Exploration Trust/Nautilus Live 2017 (CONANP, 2019), yet this species distributes only in Hawaii (Mah, 2020).

The echinoids Caenocentrotus gibbosus (Clark, 1925; Grant \& Hertlein, 1938; Maluf, 1988; Maluf,1991; Bautista-Romero et al., 1994) and Lytechinus semituberculatus (Clark, 1925; Clark,1948; Mortensen, 1943b; Caso, 1962b; Maluf, 1988; Bautista-Romero et al., 1994) have many mentions in Clarion Island, $\mathrm{REV}$, yet in all these references the locality is treated as doubtful. It is important to emphasize that both species inhabit in South America (Galápagos Islands, Perú, and Chile; Grant \& Hertlein, 1938; Solís-Marín et al., 2013a), thus their distribution in México is unlikely. Similarly, the holothuroid Holothuria (Selenkothuria) theeli is recorded from REV as doubtful (Hertlein, 1963; Maluf, 1991; Maluf \& Brusca, 2005). In the Eastern Pacific, H. (Selenkothuria) theeli inhabits in lower latitudes (i.e. Costa Rica-Perú; Solís-Marín et al., 2013a) and in México it has been collected only in Guerrero (Honey-Escandón et al., 2008). For these reasons, we determined these records as doubtful.

New records: Following review of museum material, we add 16 new records of Ophiuroidea to different states (four NAY, one COL, and one $\mathrm{MICH}$ ) and islands (two MAR, four ISA, and four REV) from the CMP (appendix 1). All the species were previously recorded in areas from the CMP (Table 1), excepting Amphiodia psara, Amphiodia tabogae, Microphiopholis geminata, and Ophiophragmus paucispinus, which represent new records for the CMP. 
Amphiodia psara has previous records in USA (California; Clark, 1935) and México (Gulf of California and Guerrero; Maluf, 1988; Maluf \& Brusca, 2005; Granja-Fernández et al., 2015b). After the taxonomic revision of A. psara in different museum collections, we did not find material from Guerrero, but for the Pacific of Baja California and Baja California Sur, the Gulf of California (Ángel de la Guarda Island, Tiburón Island, Consag Rock, and Sonora), and NAY. The records from Baja California, Baja California Sur and NAY are new, being Punta Mita, Nayarit, the southernmost area of its geographical distribution.

Amphiodia tabogae, M. geminata, and $O$. paucispinus distribute along the Eastern Pacific (México to Galápagos Islands; Solís-Marín et al., 2013a). Specifically, in México, the three species inhabit the Gulf of California; A. tabogae has been reported also in Guerrero (GranjaFernández et al., 2015b). The finding of these species in Punta Mita, NAY (A. tabogae and $M$. geminata), and in MAR (O. paucispinus), represents new records in the CMP, and fills the distribution gap along the Mexican Pacific.

Diversity and distribution: The diversity of the Echinodermata from the CMP is represented by 187 species of echinoderms belonging to 27 orders, 63 families, 110 genera, and 16 subgenera (Table 2). Compared to the total number of echinoderm species worldwide (7 437 species; WoRMS, 2020), the CMP shelters $2.5 \%$. The orders with the highest number of species in the CMP were Amphilepidida (Ophiuroidea, 40 species), Holothuriida and Dendrochirotida (Holothuroidea, 21 species, respectively), Ophiacanthida (Ophiuroidea, 16 species), and Valvatida (Asteroidea, 15 species), meanwhile the best represented families were Amphiuridae (Ophiuroidea, 26 species), and Holothuriidae (Holothuroidea, 21 species) (Table 1). On the other hand, the orders Ophiurida (45 species), Aspidochirotida (22 species), and Dendrochirotida (22 species) were the best represented in the Southern Mexican Pacific (SMP; Granja-Fernández et al. 2015b). Differences in the numbers of recorded orders between the CMP and the SMP are due to recent changes in the systematics of the Ophiuroidea (O'Hara, Stöhr, Hugall, Thuy \& Martynov, 2018) in which most of the members previously included in the order Ophiurida, now are in Amphilepidida, and in the Holothuroidea, where most of the members of Aspidochirotida were changed to Holothuriida (Miller et al., 2017). If we compare both faunas in the same classification system, similar trends are shown, suggesting that both areas possess similar faunas in terms of orders and families of echinoderms.

In the CMP, the class Ophiuroidea was the most numerous with 67 species, followed by Holothuroidea (50), Asteroidea (35), Echinoidea (32), and Crinoidea (three) (Table 2). In general, the number of species of Holothuroidea, Asteroidea and Echinoidea were similar between the CMP and the SMP, but Ophiuroidea was represented by more species in the CMP (67) than to the SMP (46); meanwhile, the class Crinoidea had not records in the SMP (Granja-Fernández et al., 2015b). Differences on the Ophiuroidea are related to the recent sampling effort directed in the

TABLE 2

Taxonomic categories of echinoderms from the Central Mexican Pacific

\begin{tabular}{|c|c|c|c|c|c|}
\hline Class & Orders & Families & Genera & Subgenera & Species \\
\hline Crinoidea & 2 & 2 & 2 & 0 & 3 \\
\hline Asteroidea & 5 & 16 & 24 & 2 & 35 \\
\hline Ophiuroidea & 6 & 20 & 35 & 4 & 67 \\
\hline Echinoidea & 8 & 14 & 23 & 0 & 32 \\
\hline Holothuroidea & 6 & 11 & 26 & 10 & 50 \\
\hline TOTAL & 27 & 63 & 110 & 16 & 187 \\
\hline
\end{tabular}


CMP (Granja-Fernández et al., 2017), and the extensive revision of samples from museum collections (reflected in the new records of species in this work). Nine species of Crinoidea from México to Perú (Solís-Marín et al., 2013a) and at least seven in the northern part of the American continent (Lambert \& Austin, 2007) are reported in the literature therefore, finding crinoids in the CMP and SMP is likely. Despite the above, a null to a scarce number of species of Crinoidea are reported in both areas. We consider that the explanation of this pattern (and in general of the Eastern Pacific) is not due to the natural biogeographic distribution of the Crinoidea in the Eastern Pacific, yet to the scarce prospection in deep waters (the natural environment of the class in the Eastern Pacific), as well as to the low interest and lack of taxonomical specialists in the class.

As previously mentioned, the CMP is represented by 187 species of echinoderms; of those, 122 inhabit in $\sim 640 \mathrm{~km}$ of the coastline of the studied states, and 142 in an extensive maritime area of the islands $\left(\sim 155000 \mathrm{~km}^{2}\right)$. The SMP has not extensive islands such as the CMP, therefore if only their coastlines are compared, the latter possesses a lower number of echinoderms species than the SMP (162 species; Granja-Fernández et al., 2015b). In order to have a better comprehension of the number of species between both areas, a standardization per area is required, therefore considering the whole CMP $(\sim 640 \mathrm{~km})$ has 5.2 species/ $\mathrm{km}$, and the SMP $(\sim 1200 \mathrm{~km})$ possesses 7.4 species $/ \mathrm{km}$. The collecting effort in more areas, habitats, and depths could explain higher biodiversity of echinoderms in the coastline of CMP, but further collections of material and biogeographic analysis are required in order to better understand their distribution pattern.

In terms of spatial distribution, the coast of the state of JAL presented the highest number of species (84), followed by the coast of NAY (74), MICH (63), and COL (55); among the islands, REV showed the major number of species (85) followed by MAR (81), MAT (48), and ISA (44) (Table 3). Compared to the most complete and recent works of echinoderms by states (Honey-Escandón et al., 2008; Rodríguez-Troncoso et al., 2013; Solís-Marín et al., 2016b; Granja-Fernández et al., 2017; Ríos-Jara et al., 2017; Nava-Bravo et al., 2019) and islands (CONANP, 2005; CONANP, 2007a; CONANP, 2007b; CONANP, 2019; Honey-Escandón et al., 2008; Solís-Marín et al., 2013a; Granja-Fernández et al., 2017), this work increases the number of recorded species in $27 \%$ for NAY, $49 \%$ for JAL, $1.5 \%$ for $\mathrm{MICH}, 70 \%$ for MAR, $64 \%$ for ISA, $48 \%$ for MAT, $38 \%$ for REV, and finally $45 \%$ for the entire CMP region. We found the same number of species for COL as its most recent account of echinoderms (Solís-Marín et al., 2016b). Differences in the numbers of species among previous studies and the present one is attributed to a more intense literature review, the validation of geographical coordinates, the correction of records, and the addition of new ones.

TABLE 3

Number of species of echinoderms from the Central Mexican Pacific

\begin{tabular}{lcccccccc} 
& NAY & JAL & COL & MICH & MAR & ISA & MAT & REV \\
Crinoidea & 0 & 0 & 2 & 0 & 1 & 0 & 0 & 0 \\
Asteroidea & 13 & 10 & 13 & 11 & 12 & 8 & 6 & 19 \\
Ophiuroidea & 33 & 27 & 18 & 12 & 39 & 13 & 16 & 25 \\
Echinoidea & 7 & 12 & 9 & 10 & 14 & 12 & 7 & 21 \\
Holothuroidea & 21 & 35 & 13 & 30 & 15 & 11 & 19 & 20 \\
TOTAL & 74 & 84 & 55 & 63 & 81 & 44 & 48 & 85 \\
\hline
\end{tabular}

$\mathrm{COL}=$ Colima ISA $=$ Isabel Island JAL $=$ Jalisco MAR $=$ Marías Islands; MAT $=$ Marietas Islands; MICH = Michoacán; $\mathrm{NAY}=$ Nayarit REV $=$ Revillagigedo Islands. 
JAL, MAR and REV were the areas with the highest number of species and have been prospected in several shallow and deep-water localities/stations since late 1800's by foreign (Albatross, Velero, and Zaca; e.g. Agassiz, 1898; Clark, 1940; Deichmann, 1958) and national expeditions (e.g. Caso, 1943b; Caso, 1961; Caso, 1962b). In contrast, the state of COL, as well as ISA and MAT islands have the lowest number of species, and their poor prospections initiated in the late 1900's (e.g. Caso, 1961; Caso, 1980), and have been mostly performed during the 2000's, strictly in shallow-waters (e.g. Chávez-Dagostino et al., 2000; Ríos-Jara et al., 2008a, Ríos-Jara et al., 2013; Sotelo-Casas et al., 2018). On the other hand, MAR and REV (the unique oceanic islands of the CMP) display unique characteristics such as particular oceanographic and topographic conditions, high productivity, mesophotic reefs, great depths, among others, which promote high levels of general marine biodiversity and endemism (CONANP, 2007a; CONANP, 2019), explaining their highest number of echinoderms in the region. In conclusion, the obtained data shows that the number of species of echinoderms in the CMP region is mostly associated with a sampling effort, yet specific conditions of particular areas (i.e. MAR and REV) can be influencing their high biodiversity, but more collections of material and further biogeographic analysis are required in order to sustain this statement.

Final considerations: The CMP is located in an oceanographic transitional zone, therefore fauna from the North and South regions of the Mexican Pacific (Arriaga-Cabrera et al., 1998), the Eastern Pacific (e.g. Solís-Marín et al., 2013a), and even of higher latitudes (e.g. Lambert \& Austin, 2007), can inhabit in the region. In this sense, MAR and REV are important as they are considered a stepping stone to import organisms from the Indo-Pacific species into the Tropical Eastern Pacific (Reyes-Bonilla \& López-Pérez, 1998), as well as in the connection for dispersal species to and from northern
(Gulf of California) and the mainland CMP (López-Pérez et al., 2015).

All the islands considered in the present work possess their own programs of management and conservation (CONANP, 2005; CONANP, 2007a; CONANP, 2007b; CONANP, 2019) in which detailed descriptions of geographical, physical, demographical, social and environmental characteristics (among others) are provided. Despite the above, we detected that all these programs underestimated the number of species of echinoderms since they reported 16 species for ISAB (CONANP, 2005), 24 for MAR (CONANP, 2007a), 25 for MAT (CONANP, 2007b), and 52 for REV (CONANP, 2019). The inclusion of all the species reported in the present work on the lists of these natural protected areas will further emphasize the importance of the islands.

Due to the importance of the islands of the CMP, most of the research of echinoderms in the region have been conducted on them (e.g. Ríos-Jara et al., 2008a; Sotelo-Casas et al., 2018) relegating the mainland of the states of NAY, JAL, COL, and MICH, which remain almost unstudied and which have a high potential of finding numerous species of echinoderms since they have complex environments (extensive bays, coastal lagoons, estuaries, mangroves and coral reefs; Arriaga-Cabrera et al., 1998). Regardless the differences on the diversity of echinoderms and exploration of the mainland and islands from the CMP, all of them are important due to 34 species collected in the region have been described as new species (five Asteroidea, 16 Ophiuroidea, five Echinoidea, and eight Holothuroidea; Table 4). This highlights the potential of the CMP in finding more new species. Although the present manuscript provides the most-completed checklist of the echinoderms in the CMP, we encourage to intensify research in shallow and deep-waters and across different habitats of all the islands and mainland of the states of the region in order to better understand its biodiversity and distributional patterns. Finally, the preservation of the marine fauna of the entire CMP is key in the maintenance of the entire Mexican and Tropical Pacific; therefore, we encourage ensuring 
TABLE 4

Described species and subspecies of Echinodermata based on material collected in the Central Mexican Pacific

\begin{tabular}{|c|c|c|c|}
\hline Class & Species & Locality & Reference \\
\hline \multirow[t]{5}{*}{ Asteroidea } & Ampheraster marianus & Marías Islands & Ludwig, 1905 \\
\hline & Astropecten regalis & San Blas, Nayarit & Gray, 1840 \\
\hline & Henricia gracilis & Marías Islands & Ludwig, 1905 \\
\hline & Henricia seminudus & Revillagigedo Islands & Clark, 1916 \\
\hline & Psilaster armatus & Marías Islands & Ludwig, 1905 \\
\hline \multirow[t]{16}{*}{ Ophiuroidea } & Amphichondrius laevis & Tenacatita Bay, Jalisco & Ziesenhenne, 1940 \\
\hline & Amphiodia sculptilis & Tenacatita Bay, Jalisco & Ziesenhenne, 1940 \\
\hline & Amphiura serpentina & Marías Islands & Lütken \& Mortensen, 1899 \\
\hline & Astrodia excavata & Marías Islands & Lütken \& Mortensen, 1899 \\
\hline & Dougaloplus notacanthus & Marías Islands & Lütken \& Mortensen, 1899 \\
\hline & Ophiacantha costata & Marías Islands & Lütken \& Mortensen, 1899 \\
\hline & Ophiacantha hirta & Marías Islands & Lütken \& Mortensen, 1899 \\
\hline & Ophiacantha moniliformis & Marías Islands & Lütken \& Mortensen, 1899 \\
\hline & Ophiacantha pyriformis & Revillagigedo Islands & Ziesenhenne, 1937 \\
\hline & Ophiernus adspersus annectens & Marías Islands & Lütken \& Mortensen, 1899 \\
\hline & Ophiolepis plateia & Tenacatita Bay, Jalisco & Ziesenhenne, 1940 \\
\hline & Ophiomitra granifera & Marías Islands & Lütken \& Mortensen, 1899 \\
\hline & Ophiomitra partita & Marías Islands & Lütken \& Mortensen, 1899 \\
\hline & Ophiophragmus lonchophorus & Tenacatita Bay, Jalisco & Ziesenhenne, 1940 \\
\hline & Ophiosphalma variabile & Marías Islands & Lütken \& Mortensen, 1899 \\
\hline & Ophiura (Ophiura) scutellata & Marías Islands & Lütken \& Mortensen, 1899 \\
\hline \multirow[t]{5}{*}{ Echinoidea } & Brisaster townsendi & Marías Islands & Agassiz, 1898 \\
\hline & Echinometra insularis & Revillagigedo Islands & Clark, 1912 \\
\hline & Encope micropora insularis & Revillagigedo Islands & Clark, 1948 \\
\hline & Hesperocidaris perplexa & Revillagigedo Islands & Clark, 1907 \\
\hline & Plexechinus cinctus & Marías Islands & Agassiz, 1898 \\
\hline \multirow[t]{8}{*}{ Holothuroidea } & Cucumaria flamma & Colima, Nayarit, Marietas Islands & $\begin{array}{l}\text { Solís-Marín \& Laguarda- } \\
\text { Figueras, } 1999\end{array}$ \\
\hline & Lissothuria imbricata & Jalisco & Solís-Marín et al., 2018 \\
\hline & Holothuria (Selenkothuria) carere & Michoacán & Honey-Escandón et al., 2011 \\
\hline & $\begin{array}{l}\text { Holothuria }(\text { Selenkothuria) } \\
\text { portovallartensis }\end{array}$ & Puerto Vallarta, Jalisco & Caso, 1954 \\
\hline & Psolus pauper & Marías Islands & Ludwig, 1894 \\
\hline & Thyone bidentata & Tenacatita Bay, Jalisco & Deichmann, 1941 \\
\hline & Thyone parafusus & Tenacatita Bay, Jalisco & Deichmann, 1941 \\
\hline & Ypsilothuria bitentaculata & Marías Islands & Ludwig, 1894 \\
\hline
\end{tabular}

its effective protection through inventories of marine and coastal species.

Ethical statement: authors declare that they all agree with this publication and made significant contributions; that there is no conflict of interest of any kind; and that we followed all pertinent ethical and legal procedures and requirements. All financial sources are fully and clearly stated in the acknowledgements section. A signed document has been filed in the journal archives. 


\section{ACKNOWLEDGMENTS}

We are very grateful to Alicia Durán, Alejandra Martínez-Melo, and Pedro Medina for providing valuable literature. We wish to express our sincere gratitude to Alicia Durán (ICML), Penny Benson and Adam Baldinger (MCZ), Gordon Hendler and Cathy Groves (LACM) for providing their support during collection's visits. This work was supported by a scholarship from CONACYT 336853, and an Ernst Mayr Grant (MCZ), and is part of the post-doc of RGF at UdG supported by PRODEP (511-6/2019.-12278). We thank four anonymous reviewers and editor for commenting and improving the manuscript.

\section{RESUMEN}

\section{Equinodermos (Echinodermata) del Pacífico Central mexicano}

Introducción: Los equinodermos del Pacífico Central mexicano poseen un alto interés científico, pero anterior al presente trabajo existía un vacío en la información básica como listados de especies incompletos con inconsistencias sistemáticas y de distribución espacial. Objetivo: Proporcionar una revisión histórica y un listado de especies actualizado con la riqueza completa de equinodermos para cada estado e islas de la región. Métodos: Se elaboró un listado de especies basado en una búsqueda exhaustiva de Echinodermata, y fue complementada con la revisión taxonómica de Ophiuroidea de colecciones científicas. Todas las coordenadas geográficas de los registros fueron validadas. Resultados: La región posee 187 especies de Echinodermata: tres Crinoidea, 35 Asteroidea, 67 Ophiuroidea, 32 Echinoidea, y 50 Holothuroidea. Detectamos 52 registros en la literatura considerados como inválidos y cinco como dudosos. Proveemos 16 nuevos registros de Ophiuroidea para diversos estados e islas; de estos, cuatro son nuevos registros para la región. Jalisco presentó el mayor número de especies (84), seguido por las costas de Nayarit (74), Michoacán (63), y Colima (55); entre las islas, Revillagigedo presentó el mayor número de especies (85) seguido por Marías (81), Marietas (48), e Isabel (44). Conclusiones: Los números de especies de la región están mayormente relacionados tanto con el esfuerzo de muestreo como con características ambientales que promueven una alta biodiversidad. El Pacífico Central mexicano es una región oceanográfica con condiciones mixtas del norte y sur del Pacífico mexicano, y por lo tanto con una importancia biogeográfica reflejada en la riqueza de la región.

Palabras clave: Echinodermata; biodiversidad; listado de especies; revisión histórica; nuevos registros.

\section{REFERENCES}

Numbers in brackets refer to literature where the taxa were recorded (Table 1)

Adem, J., Cobo, E., Blásquez, L., Miranda, F., Villalobos, A., Herrera, T., . . V Vázquez, L. (1960). La Isla Socorro. Archipiélago de Revillagigedo. Monografias del Instituto de Geofisica. Universidad Nacional Autónoma de México, 2, 1-234. [1]

Agassiz, A. (1872). Revision of the Echini. Part I. Illustrated catalogue of the Museum of Comparative Zoölogy at Harvard College, 7, 1-242. [2]

Agassiz, A. (1898). Reports on the dredging operations off the West coast of Central America to the Galapagos, to the West coast of Mexico, and in the Gulf of California, in charge of Alexander Agassiz, carried on by the U.S. Fish Commission Steamer "Albatross", during 1891, Lieut. Commander Z.L. Tanner, U.S.N., commanding. XXIII. Preliminary report on the Echini. Bulletin of the Museum of Comparative Zoölogy at Harvard College, 32(5), 71-86. [3]

Agassiz, A. (1904). Reports on an exploration off the West coast of Mexico, Central and South America and off the Galapagos Islands, in charge of Alexander Agassiz by the U.S. Fish Commission Steamer "Albatross", during 1891, lieut. Commander Z.L. Tanner, U.S.N commanding. XXXII. The Panamic deep sea Echini. Memoirs of the Museum of Comparative Zoology at Harvard College, 31, 1-243. [4]

Alvarado, J.J., Reyes-Bonilla, H., \& Benítez-Villalobos, F. (2015). Diadema mexicanum, erizo de mar clave en los arrecifes coralinos del Pacífico Tropical Oriental: lo que sabemos y perspectivas futuras (Diadematoida: Diadematidae). Revista de Biología Tropical, 63(2), 135-157. [5]

Arriaga-Cabrera, L., Vázquez-Domínguez, E., GonzálezCano, J., Jiménez-Rosenberg, R., Muñoz-López, E., \& Aguilar-Sierra, V. (1998). Regiones marinas prioritarias de México. México: Comisión Nacional para el Conocimiento y uso de la Biodiversidad.

Ayala-Bocos, A., Reyes-Bonilla, H., Herrero-Pérezrul, M.D., Walther-Mendoza, M., \& Castañeda-Fernández de Lara, V. (2011). New records and range extensions of Astrodictyum panamense (Ophiuroidea: Gorgonocephalidae) in the eastern Pacific Ocean. Marine Biodiversity Records, 4(46), 1-3. [6]

Bautista-Romero, J., Reyes-Bonilla, H., Lluch-Cota, D.B., \& Lluch-Cota S.E (1994). Aspectos generales de la fauna marina. In A. Ortega-Rubio \& A. CastellanosVera (Eds.), La Isla Socorro, Reserva de la Biósfera Archipiélago de Revillagigedo, México (pp. 247-275). La Paz, México: Centro de Investigaciones Biológicas del Noroeste S.C. [7] 
Borrero-Pérez, G.H., \& Vanegas-González, M.J. (2019) Holothuria (Mertensiothuria) viridiaurantia sp. nov. (Holothuriida, Holothuriidae), a new sea cucumber from the Eastern Pacific Ocean revealed by morphology and DNA barcoding. ZooKeys, 893, 1-19.

Caso, M.E. (1943). Contribución al conocimiento de los astéridos de México (Master's thesis). Universidad Nacional Autónoma de México, México. [8]

Caso, M.E. (1948a). Contribución al conocimiento de los equinoideos de México. II. Algunas especies de equinoideos litorales. Anales del Instituto de Biología, Universidad Nacional Autónoma de México, 19(1), 183-231. [9]

Caso, M.E. (1948b). Datos históricos y estado actual de los conocimientos de la fauna de astéridos de México. Sociedad Mexicana de Historia Natural, 1, 21-32. [10]

Caso, M.E. (1951). Contribución al conocimiento de los ofiuroideos de México. I. Algunas especies de ofiuroideos litorales. Anales del Instituto de Biología, Universidad Nacional Autónoma de México, 22(1), 219-312. [11]

Caso, M.E. (1954). Contribución al conocimiento de los holoturoideos de México. Algunas especies de holoturoideos litorales y descripción de una nueva especie Holothuria portovallartensis. Anales del Instituto de Biología, Universidad Nacional Autónoma de México, 25, 417-422. [12]

Caso, M.E. (1961). Los Equinodermos de México (Doctoral thesis). Universidad Nacional Autónoma de México, México. [13]

Caso, M.E. (1962a). Estudios sobre Astéridos de México. Observaciones sobre especies pacíficas del género Acanthaster y descripción de una subespecie nueva, Acanthaster ellisii pseudoplanci. Anales del Instituto de Biología, Universidad Nacional Autónoma de México, 32(1-2), 313-331. [14]

Caso, M.E. (1962b). Estudios sobre equinodermos de México. Contribución al conocimiento de los equinodermos de las Islas Revillagigedo. Anales del Instituto de Biología, Universidad Nacional Autónoma de México, 33(1-2), 293-330. [15]

Caso, M.E. (1965). Estudios sobre equinodermos de México. Contribución al conocimiento de los holoturoideos de Zihuatanejo y de la Isla de Ixtapa (primera parte). Anales del Instituto de Biología, Universidad Nacional Autónoma de México, 36, 253-291. [16]

Caso, M.E. (1966). Contribución al estudio de los holoturoideos de México. Morfología interna y ecología de Stichopus fuscus Ludwig. Anales del Instituto de Biología, Universidad Nacional Autónoma de México, 37, 175-182. [17]
Caso, M.E. (1974a). Contribución al estudio de los equinoideos de México. Morfología de Tripneustes depressus Agassiz y estudio comparativo entre $T$. ventricosus y T. depressus. Anales del Instituto de Biología, Universidad Nacional Autónoma de México, 1(1), 25-40. [18]

Caso, M.E. (1974b). Morfología externa de Acanthaster planci (Linnaeus). Symposium on Indian Ocean and Adjacent Seas. Journal of the Marine Biological Association of India, 16(1), 83-93. [19]

Caso, M.E. (1978a). Ciencia y técnica de los equinodermos en relación con el hombre. Primera parte. Aspecto científico. Anales del Centro de Ciencias del Mar y Limnolología, Universidad Nacional Autónoma de México, 5(1), 255-286. [20]

Caso, M.E. (1978b). Los equinoideos del Pacífico de México. Parte primera. Órdenes Cidaroidea y Aulodonta. Parte segunda. Órdenes Stiridonta y Camarodonta. Publicaciones Especiales, Centro de Ciencias del Mar y Limnología, Universidad Nacional Autónoma de México, 1, 1-244. [21]

Caso, M.E. (1979a). Los equinodermos de la Bahía de Mazatlán, Sinaloa. Anales del Centro de Ciencias del Mar y Limnología, Universidad Nacional Autónoma de México, Publicación Especial, 6(1), 197-368. [22]

Caso, M.E. (1979b). Los equinodermos (Asteroidea, Ophiuroidea y Echinoidea) de la Laguna de Términos, Campeche. Centro de Ciencias del Mar y Limnología, Universidad Nacional Autónoma de México, Publicación Especial, 3, 1-186. [23]

Caso, M.E. (1980). Los equinoideos del Pacífico de México. Parte tercera. Orden Clypeasteroida. Publicaciones Especiales, Centro de Ciencias del Mar y Limnología, Universidad Nacional Autónoma de México, 4, 1-252. [24]

Caso, M.E. (1983a). Especies del género Amphichondrius de la costa del Pacífico Americano. Descripción de una nueva especie del género Amphichondrius. Amphichondrius unamexici sp. nov. (Ophiuroidea). Anales del Instituto de Ciencias del Mar y Limnología, Universidad Nacional Autónoma de México, 10(1), 209-230. [25]

Caso, M.E. (1983b). Los equinoideos del Pacífico de México. Parte cuarta. Órdenes Cassiduloida y Spatangoida. Publicaciones Especiales, Centro de Ciencias del Mar y Limnología, Universidad Nacional Autónoma de México, 6, 1-200. [26]

Caso, M.E. (1986a). Descripción de una nueva especie de ofiuroideo de la Bahía de Mazatlán, Sin. Ophioderma sodipallaresi sp. nov. y comparación con Ophioderma variegatum Lütken. Anales del Instituto de Ciencias del Mar y Limnología, Universidad Nacional Autónoma de México, 13(2), 223-248. [27] 
Caso, M.E. (1986b). Los equinodermos del Golfo de California colectados en las campañas SIPCO I-II-III a bordo del B/O "El Puma". Anales del Instituto de Ciencias del Mar y Limnología, Universidad Nacional Autónoma de México, 13(1), 91-184. [28]

Caso, M.E. (1992). Los equinodermos (Asteroideos, ofiuroideos y equinoideos) de la Bahía de Mazatlán, Sinaloa. Anales del Centro de Ciencias del Mar y Limnología, Universidad Nacional Autónoma de México, Publicación Especial, 11, 1-214. [29]

Caso, M.E. (1994). Estudio morfológico, taxonómico, ecológico y distribución geográfica de los asteroideos colectados durante las campañas oceanográficas Cortés 1, 2, 3. Anales del Instituto de Ciencias del Mar y Limnología, Universidad Nacional Autónoma de México, Publicación Especial, 12, 1-111. [30]

Chacón-Torres, A., Villaroel-Melo, M., Arceo-Zataray, M., Carrillo-Orea, M.P., Corona-García J.M, Chavarría-Guillén, M.P., . . . Villegas-Sandoval, R.E. (1993). Reconocimiento ecológico de la Isla Socorro, Archipiélago Revillagigedo, Colima, México. Revista Trimestral de Ciencia, Arte y Cultura, Universidad Michoacana, 9, 11-30. [31]

Chan, G.L. (1974). Report on biological observations of the Revillagigedos expeditions. Kentfield, USA: NAUI Bio-marine Exploration Seminar, College of Marin. [32]

Chávez-Dagostino, R.M., Nepote-González, A.C., SolísMarín, F.A., \& Medina-Rosas, P. (2000). Listado preliminar sobre equinoideos y asteroideos (Echinodermata: Echinoidea y Asteroidea) de las Islas Marietas, Nayarit, México. Mexico, 2(1), 69-72. [33]

Clark, A.H. (1916). Six new starfishes from the Gulf of California and adjacent waters. Proceedings of the Biological Society of Washington, 29, 51-62. [34]

Clark A.M. (1970). Notes on the family Amphiuridae (Ophiuroidea). Bulletin of the British Museum (Natural History) Zoology, 19(1), 1-81. [35]

Clark, H.L. (1907). The Cidaridae. Bulletin of the Museum of Comparative Zoölogy, 51(7), 165-230. [36]

Clark, H.L. (1912). Hawaiian and other Pacific Echini. The Pedinidae, Phymosomatidae, Stomopneustidae, Echinidae, Temnopleuridae, Strongylocentrotidae, and Echinometridae. Memoirs of the Museum of Comparative Zoölogy at Harvard College, 34(4), 209-383. [37]

Clark, H.L. (1915). Catalogue of recent ophiurans: based on the collection of the Museum of Comparative Zoölogy. Memoirs of the Museum of Comparative Zoölogy at Harvard College, 25(4), 164-376. [38]
Clark, H.L. (1925). A catalogue of the recent sea-urchins (Echinoidea) in the collection of the British Museum (Natural History). London, United Kingdom: The British Museum (Natural History).

Clark, H.L. (1935). Some new echinoderms from California. The Annals and Magazine of Natural History, 10(15), 120-129.

Clark, H.L. (1940). XXI. Notes on Echinoderms from the West Coast of Central America. Eastern Pacific Expeditions of the New York Zoological Society. Zoologica, 25(22), 331-352. [39]

Clark, H.L. (1948). A report on the Echini of the warmer Eastern Pacific, based on the collections of the Velero III. Allan Hancock Pacific Expeditions, 8(5), 225 360. [40]

CONANP. (2004). Programa de conservación y manejo Reserva de la Biosfera Archipiélago de Revillagigedo, México. Ciudad de México, México: Comisión Nacional de Áreas Naturales Protegidas, Secretaría de Medio Ambiente y Recursos Naturales. [41]

CONANP. (2005). Programa de conservación y manejo del Parque Nacional Isla Isabel, México. Ciudad de México, México: Comisión Nacional de Áreas Naturales Protegidas, Secretaría de Medio Ambiente y Recursos Naturales. [42]

CONANP. (2007a). Programa de conservación y manejo, Reserva de la Biosfera Islas Marías, México. Ciudad de México, México: Comisión Nacional de Áreas Naturales Protegidas, Secretaría de Medio Ambiente y Recursos Naturales. [43]

CONANP. (2007b). Programa de conservación y manejo, Parque Nacional Islas Marietas, México. Ciudad de México, México: Comisión Nacional de Áreas Naturales Protegidas, Secretaría de Medio Ambiente y Recursos Naturales. [44]

CONANP. (2019). Programa de manejo, Parque Nacional Revillagigedo. Ciudad de México, México: Comisión Nacional de Áreas Naturales Protegidas, Secretaría de Medio Ambiente y Recursos Naturales. [45]

Deichmann, E. (1937). The Templeton Crocker Expedition. IX. Holothurians from the Gulf of California, the West Coast of Lower California and Clarion Island. Zoologica, 22, 161-176. [46]

Deichmann, E. (1941). The Holothuroidea collected by the Velero III during the years 1932 to 1938. Part I, Dendrochirota. Allan Hancock Pacific Expeditions, 8, 61-195. [47]

Deichmann, E. (1958). The Holothuroidea collected by the Velero III and IV during the years 1932 to 1954 . Part 
II Aspidochirota. Allan Hancock Pacific Expeditions, 11(2), 253-348. [48]

Devaney, D.M. (1970). Studies on Ophiocomid brittlestars. I. A new genus (Clarkcoma) of Ophiocominae with a reevaluation of the genus Ophiocoma. Smithsonian Contributions to Zoology, 51, 1-41. [49]

Döderlein, L. (1911). Beiträge zur Naturgeschichte Ostasiens. Über Japanische und andere Euryalae. Abhandlugen der Bayerischen Akademie der Wissencaften. Mathemaatisch-Naturwissenschaftliche Abteilung, 2(5), 1-123. [50]

Downey, M.E. (1968). Catalog of recent Echinoid type specimens in the U.S. National Museum Smithsonian Institution and the Museum of Comparative Zoology Harvard University. United States National Museum Bulletin, 264, 1-99. [51]

Downey, M.E. (1969). Catalog of recent ophiuroid type specimens in major collections in the United States. United States National Museum Bulletin, 293, 1-239. [52]

Espino-Barr, E., Cibrián-Rodríguez, R., \& García-Boa, A. (1996). Estructura y densidad de la población del erizo tinta Diadema mexicanum en el litoral rocoso del estado de Colima. Ciencia Pesquera, 12, 60-67. [53]

Fisher, W.K. (1911). Asteroidea of the North Pacific and adjacent waters. Part 1. Phanerozonia and Spinulosa. Smithsonian Institution, United States National Museum Bulletin, 76, 1-419. [54]

Fisher, W.K. (1928a). Asteroidea of the North Pacific and adjacent waters. Part 2. Forcipulata (part). Smithsonian Institution, United States National Museum Bulletin, 76, 1-245. [55]

Fisher, W.K. (1928b). Asteroidea of the North Pacific and adjacent waters. Part 3. Forcipulata (concluded). Smithsonian Institution, United States National Museum Bulletin, 76, 1-356. [56]

Frontana-Uribe, S.C., Hernández-Alcántara, P., \& SolísWeiss, V. (2000). Ophiocomella schmitti (Echinodermata: Ophiuroidea) from Socorro Island, Mexican Pacific. Revista de Biología Tropical, 49(2), 766. [57]

Fuentes-Farías, A.M., Villarroel-Melo, M.D., \& SolísMarín, F.A. (2005). Los invertebrados marinos. Anexo 4.20 y 4.21. Listado de especies de equinodermos del Estado de Michoacán. In L.E. Villaseñor-Gómez (Ed.), La biodiversidad en Michoacán, Estudio de Estado (pp. 85-88). México: Comisión Nacional para el Conocimiento y Uso de la Biodiversidad, Secretaría de Urbanismo y Medio Ambiente, Universidad Michoacana de San Nicolás de Hidalgo. [58]
García-Hernández, V.C., Reyes-Bonilla, H., Balart, E.F., Ríos-Jara, E., Lluch-Cota, S.E., \& Serviere-Zaragoza, E. (2014). Comparison of ecological diversity and species composition of macroalgae, benthic macroinvertebrates, and fish assemblages between two tropical rocky reefs. Revista de Biología Marina y Oceanografia, 49, 477-491. [59]

Granja-Fernández, R., Antonio-Pérez, V., \& López-Pérez, R. A. (2013a). Euapta godeffroyi (Holothuroidea: Synaptidae): Filling the distribution gap between Mexico and Costa Rica, eastern tropical Pacific. Hidrobiológica, 23(1), 130-132. [60]

Granja-Fernández, R., Hernández-Moreno, P., \& BastidaZavala, R. (2013b). First record of the association between Malmgreniella cf. variegata (Polychaeta, Polynoidae) and Ophionereis annulata (Echinodermata, Ophionereididae) in the Mexican Pacific. Symbiosis, 60, 85-90. [61]

Granja-Fernández, R., Herrero-Pérezrul, M.D., LópezPérez, R.A., Hernández, L., Rodríguez-Zaragoza, F.A., Jones, R.W., \& Pineda-López, R. (2014). Ophiuroidea (Echinodermata) from coral reefs in the Mexican Pacific. Zookeys, 406, 101-145. [62]

Granja-Fernández, R., Herrero-Pérezrul, M.D., LópezPérez, R.A., Hernández-Morales, A., \& Rangel-Solís, P.D. (2015a). A literature review of the Ophiuroidea (Echinodermata) from the Pacific coast of Mexico. Revista de Biología Tropical, 63(2), 37-47. [63]

Granja-Fernández, R., Solís-Marín, F.A., Benítez-Villalobos, F., Herrero-Pérezrul, M.D., \& López-Pérez, A. (2015b). Checklist of echinoderms (Echinodermata) from the Southern Mexican Pacific: a historical review. Revista de Biología Tropical, 63(2), 87-114.

Granja-Fernández, R., Rodríguez-Troncoso, A.P., HerreroPérezrul, M.D., Sotelo-Casas, R.C., Flores-Ortega, J.R., Godínez-Domínguez, E., . . . Cupul-Magaña, A.L. (2017). Ophiuroidea (Echinodermata) from the Central Mexican Pacific: an updated checklist including new distribution records. Marine Biodiversity, 47, 167-177. [64]

Grant, U.S., \& Hertlein, L.G. (1938). The West American Cenozoic Echinoidea. Publications of the University at Los Angeles in Mathematical and Physical Sciences, 2, 1-225. [65]

Gray, J.E. (1840). XXII. A synopsis of the genera and species of the class Hypostoma (Asterias, Linnaeus). The Annals and Magazine of Natural History, 6, 175-184. [66]

Harold, A.S., \& Telford, M. (1990). Systematics, phylogeny and biogeography of the genus Mellita (Echinoidea: Clypeasteroida). Journal of Natural History, 24(4), 987-1026. [67] 
Hartlaub C. (1895). Reports on the dredging operations off the west coast of Central America to the Galapagos, to the west coast of Mexico, and in the Gulf of California, in charge of Alexander Agassiz, carried on by the U. S. Fish Commission steamer "Albatross", during 1891, Lieut. Commander Z. L. Tanner, U. S. N., commanding. 13. Die Comatuliden. Bulletin of the Museum of Comparative Zoölogy at Harvard College, 27, 129-152. [68]

Haszprunar, G., \& Spies, M. (2014). An integrative approach to the taxonomy of the crown-of-thorns starfish species group (Asteroidea: Acanthaster): A review of names and comparison to recent molecular data. Zootaxa, 3841, 271-284. [69]

Hendler, G. (1996). Taxonomic atlas of The Benthic Fauna of the Santa Maria Basin and Western Santa Barbara Channel. Class Ophiuroidea (Vol. 14). Santa Barbara, USA: Santa Barbara Museum of Natural History. [70]

Hendler, G. (2011). New light on the nomenclature, taxonomy, and biology of Hemipholis species (Echinodermata: Ophiurodea: Ophiactidae). Zootaxa, 3048, 44-52. [71]

Hendler, G., Miller, J.E., Pawson, D.L., \& Kier, P.M. (1995). Sea stars, sea urchins, and allies. Echinoderms of Florida and the Caribbean. Washington, USA: Smithsonian Institution.

Hermosillo-Nuñez, B.B., Rodríguez-Zaragoza, F.A., Ortiz, M., Galván-Villa, C., Cupul-Magaña, A., \& RíosJara, E. (2015). Effect of habitat structure on the most frequent echinoderm species inhabiting coral reef communities at Isla Isabel National Park (Mexico). Community Ecology, 16(1), 125-134. [72]

Hermosillo-Nuñez, B.B., Rodríguez-Zaragoza, F.A., Ortiz, M., Calderon-Aguilera, L.E., Cupul-Magaña, A.L. (2016). Influence of the coral reef assemblages on the spatial distribution of echinoderms in a gradient of human impacts along the tropical Mexican Pacific. Biodiversity and Conservation, 25, 2137-2152. [73]

Herrero-Pérezrul, M.D., Reyes-Bonilla, H., GonzálezAzcárraga A., Cintra-Buenrostro, C.E., \& RojasSierra, A. (2007). Equinodermos. In G.D. Danemann \& E. Ezcurra (Eds.), Bahía de los Ángeles: recursos naturales y comunidad, Línea base 2007 (pp. 339361). México: Secretaría de Medio Ambiente y Recursos Naturales, Instituto Nacional de Ecología, Pronatura Noroeste A.C., San Diego Natural History Museum. [74]

Herrero-Pérezrul, M.D., Ramírez-Ortiz, G., RosalesEstrada, M., \& Reyes-Bonilla, H. (2015). Densidad poblacional y distribución espacial de erizos de mar (Echinodermata: Echinoidea) en Isla Socorro, Archipiélago de Revillagigedo, México. Revista de Biología Tropical, 63(2), 221-232. [75]
Herrero-Pérezrul, M.D., Reyes-Bonilla, H., \& Granja-Fernández, R. (2017). Effects of environmental factors on the abundances of the basket stars Astrocaneum spinosum and Astrodictyum panamense (Ophiuroidea: Gorgonocephalidae) in the northern Gulf of California, Mexico. Marine Biology Research, 13(2), 210-219. [76]

Hertlein, L.G. (1963). Contribution to the biogeography of Cocos Island, including a bibliography. Proceedings of the California Academy of Sciences, 32(8), 219289. [77]

Hickman, C.P. (1998). A field guide to sea stars and other echinoderms of Galápagos. Virginia, USA: Sugar Spring Press. [78]

Holguín-Quiñones, O.E. (1994). Comunidades bentónicas marinas. In A. Ortega-Rubio \& A. Castellanos-Vera (Eds.), La Isla Socorro, Reserva de la Biósfera Archipiélago de Revillagigedo, México (pp. 225-245). La Paz, México: Centro de Investigaciones Biológicas del Noroeste, S.C. [79]

Holguin-Quiñones, O.E., \& Michel-Morfín, J.E. (2006). Population structure and accompanying biota of the snail Turbo (Callopoma) funiculosus (Gastropoda: Turbinidae), on Socorro Island, Revillagigedo Archipielago, Mexico. Revista de Biología Tropical, 54(4), 1079-1084. [80]

Honey-Escandón, M., Solís-Marín F.A., \& LaguardaFigueras A. (2008). Equinodermos (Echinodermata) del Pacífico Mexicano. Revista de Biología Tropical, 56(3), 57-73. [81]

Honey-Escandón M., Solís-Marín, F.A., \& LaguardaFigueras, A. (2011). Holothuria (Selenkothuria) carere, a new species of sea cucumber (Echinodermata: Holothuroidea) from the Mexican Pacific. Zootaxa, 2922, 27-33. [82]

Koehler, R. (1907). Révision de la Collection des Ophiures du Museum D'Histoire Naturelle de Paris. Bulletin Scientifique de la France et de la Belgique, 61, 279370. [83]

Kroh, A., \& Mooi, R. (2020). World Echinoidea Database. Retrieved from http://www.marinespecies.org/echinoidea on 2020-06-13

Lambert, P., \& Austin, W. (2007). Brittle stars, sea urchins and feather stars of British Columbia, Southeast Alaska and Puget Sound. Victoria, Canada: Royal BC Museum Handbook.

López-Pérez, A., Cupul-Magaña, A., Ahumada-Sempoal, M., Medina-Rosas, P., Reyes-Bonilla, H., HerreroPérezrul, M.D., . . . Lara-Hernández, J. (2015). The coral communities of the Islas Marias archipelago, Mexico: structure and biogeographic relevance to the Eastern Pacific. Marine Ecology, 37, 679-690. 
López-Uriarte, E., Ríos-Jara, E., Galván-Villa, C.M., Juárez-Carrillo, E., Enciso-Padilla, I., Robles-Jarero, E.G., \& Pérez-Peña, M. (2009). Macroinvertebrados bénticos del litoral somero de Punta La Rosada, Bahía Chamela, Jalisco. Scientia-CUCBA, 11(1-2), 57-68. [84]

Ludwig, H. (1893). Vorläufiger Bericht über die erbeuteten Holothurien. Reports on the Dredging Operations off the West Coast of Central America to the Galapagos, etc., by the U. S. Fish Commission Steamer "Albatross". IV. Bulletin of the Museum of Comparative Zoology at Harvard College, 24(4), 105-114.

Ludwig, H. (1894). Reports on an exploration off the west coasts of Mexico, central and South America, and off the Galapagos Islands, in charge of Alexander Agassiz, by the U.S. Fish Commission Steamer "Albatross" during 1891, Lieut. Commander Z.L. Tanner, U.S.N., commanding. XII. The Holothurioidea. Memoirs of the Museum of Comparative Zoölogy at Harvard College, 17(3), 1-183. [85]

Ludwig, H. (1905). VII. Asteroidea. Reports on an exploration off the West Coast of Mexico, Central and South America, and off the Galapagos islands in charge of Alexander Agassiz, by the U.S. Fish Commission steamer "Albatross", during 1891, lieut. Commander Z.L. Tanner, U.Sn., commanding. XXXV. Reports on the scientific results of the expedition to the Tropical Pacific, in charge of Alexander Agassiz on the U.S. Fish Commission Steamer "Albatross", from August 1899, to March, 1900, commander Jefferson F. Moser, U.S.N., commanding. Memoirs of the Museum of Comparative Zoölogy at Harvard College, 32, 1-292. [86]

Luke, S.R. (1982). Catalog of the benthic invertebrate collections of the SCRIPPS Institution of Oceanography, Echinodermata. California, USA: SCRIPPS Institution of Oceanography Reference Series, no. 82-5, University of California. [87]

Lütken, C.F., \& Mortensen, T. (1899). The Ophiuridae. Memoirs of the Museum of Comparative Zoology, 23, 93-208. [88]

Madsen, F.J. (1977). The Ophioleucidae (Ophiuroidea). Galathea Report. Scientific Results of the Danish Deep-Sea Expedition Round the world 1950-52, 14, 109-122. [89]

Mah, C.L. (2020). World Asteroidea Database. Retrieved from http://www.marinespecies.org/asteroidea

Maluf, L.Y. (1988). Composition and distribution of the Central Eastern Pacific echinoderms. Natural History Museum of Los Angeles County. Technical Report, 2, 1-242. [90]

Maluf, L.Y. (1991). Echinoderm fauna of the Galápagos Islands. In M.J. James (Ed.), Galápagos marine invertebrates: taxonomy, biogeography, and evolution in Darwin's Islands (pp. 345-367). New York, USA: Plenum press. [91]

Maluf, L.Y., \& Brusca, R.C. (2005). Echinodermata. In M.E. Hendrickx, R.C. Brusca, \& L.T. Findley (Eds.), $A$ distributional checklist of the macrofauna of the Gulf of California, Mexico. Part I. Invertebrates (pp. 327-343). Tucson, USA: Arizona-Sonora Desert Museum. [92]

Martínez-Melo, A., Solís-Marín, F.A., Buitrón-Sánchez, B.E., \& Laguarda-Figueras, A. (2015). Taxonomía y biogeografía ecológica de los equinoideos irregulares (Echinoidea: Irregularia) de México. Revista de Biología Tropical, 63(2), 59-75. [93]

Massin, C., \& Hendrickx, M.E. (2011). Deep-water Holothuroidea (Echinodermata) collected during the TALUD cruises off the Pacific coast of Mexico, with the description of two new species. Revista Mexicana de Biodiversidad, 82, 413-443. [94]

McCartney, M.A., Keller, G., \& Lessios, H.A. (2000). Dispersal barriers in tropical oceans and speciation in Atlantic and eastern Pacific sea urchins of the genus Echinometra. Molecular Ecology, 9, 1391-1400. [95]

Messing, C. (2020). World List of Crinoidea. Crinoidea. Retrieved from http://www.marinespecies.org/aphia. php?p=taxdetails\&id=123081 on 2020-06-13

Miller, A.K., Kerr, A.M., Paulay, G., Reich, M., Wilson, N.G., Carvajal, J.I., \& Rouse, G.W. (2017). Molecular phylogeny of extant Holothuroidea (Echinodermata). Molecular Phylogenetics and Evolution, $111,110-131$.

Miranda, A., Ambriz, G., Valencia, D., Sánchez, M., \& Szekely, A. (2011). Programa de Manejo del santuario de las Islas de la Bahía de Chamela: Islas La Pajarera, Cocinas, Mamut, Colorada, San Pedro, San Agustín, San Andrés y Negrita, e islotes Los Anegados, Novillas, Mosca y Submarino, Jalisco, México. Ciudad de México, México: Comisión Nacional de Áreas Naturales Protegidas. [96]

Mooi, R., Constable H., Lockhart, S., \& Pearse, J. (2004). Echinothurioid phylogeny and the phylogenetic significance of Kamptosoma (Echinoidea: Echinodermata). Deep-Sea Research II, 51, 1903-1919.

Mortensen, T. (1928). A monograph of the Echinoidea I. Cidaroidea. Copenhagen, Denmark: C.A. Rietzel publisher, Oxford University press. [97]

Mortensen, T. (1940). A monograph of the Echinoidea. III. 1. Aulodonta. With additions to Vol. II (Lepidocentroida and Stirodonta). Copenhagen, Denmark: C.A. Reitzel publisher. [98] 
Mortensen, T. (1943a). A monograph of the Echinoidea. III. 2. Camarodonta. I. Orthopsidae, Glyphocyphidae, Temnopleuridae and Toxopneustidae. Copenhagen, Denmark: C.A. Reitzel publisher. [99]

Mortensen, T. (1943b). A monograph of the Echinoidea. III. 3. Camarodonta. II. Echinidae, Strongylocentrotidae, Parasaleniidae, Echinometridae. Copenhagen, Denmark: C.A. Reitzel publisher. [100]

Mortensen, T. (1948a). A monograph of the Echinoidea. IV. 1. Holectypoida, Cassiduloida. Copenhagen, Denmark: C.A. Reitzel publisher. [101]

Mortensen, T. (1948b). A monograph of the Echinoidea. IV. 2. Clypeastroida. Clypeastridae, Arachnoididae, Fibulariidae, Laganidae and Scutellidae. Copenhagen, Denmark: C.A. Reitzel publisher. [102]

Mortensen, T. (1951). A monograph of the Echinoidea. V. 2. Spatangoida II. Amphisternata, II. Spatangidae, Loveniidae, Pericosmidae, Schizasteridae, Brissidae. Copenhagen, Denmark: C.A. Reitzel publisher. [103]

Nava-Bravo, H.H., Solís-Marín, F.A., Fuentes-Farías, A.L., Cruz-Barraza, J.A., Bastida-Zavala, J.R., LaguardaFigueras, A., ... Conejeros-Vargas, C.A. (2019). Invertebrados marinos. Apéndice 35. In A. CruzAngón, K.C. Nájera-Cordero, \& E.D. Melgarejo (Coords.), La biodiversidad en Michoacán. Estudio de Estado 2, vol. II (pp. 291-306). México: Comisión Nacional para el Conocimiento y Uso de la Biodiversidad. [104]

Nepote-González, A.C. (1998). Holoturias (Echinodermata: Holothuroidea) de las islas Marietas, Bahía de Banderas, Jalisco-Nayarit, México (Bachelor thesis). Universidad de Guadalajara, México. [105]

O'Hara, T.D., Stöhr, S., Hugall, A.F., Thuy, B., \& Martynov, A. (2018). Morphological diagnoses of higher taxa in Ophiuroidea (Echinodermata) in support of a new classification. European Journal of Taxonomy, $416,1-35$.

Okanishi, M., \& Fujita, T. (2014). A taxonomic review of the genus Astrodia (Echinodermata: Ophiuroidea: Asteronychidae). Journal of the Marine Biological Association of the United Kingdom, 94(1), 187-201. [106]

Padilla-Pérez, M.S., Rodríguez-Troncoso A.P., SoteloCasas R.C., \& Cupul-Magaña A.L. (2017). Equinodermos del Parque Nacional Islas Marietas: Generalidades, importancia e identificación visual como herramienta para su protección. Áreas Naturales Protegidas Scripta, 3(2), 51-92. [107]

Parker, R.H. 1963. Zoogeography and ecology of some macro-invertebrates, particularly mollusks, in the Gulf of California and continental slope off Mexico. Massachusetts, USA: Woods Hole.
Pawson, D. (1983). Psychronaetes hanseni, a new genus and species of Elasipodan sea cucumber from the Eastern Central Pacific (Echinodermata: Holothuroidea). Proceedings of the Biological Society of Washington, 96(1), 154-159.

Reyes-Bonilla, H. (1995). Asteroidea and Echinoidea (Echinodermata) of Isla San Benedicto, Revillagigedo Archipelago, México. Revista de Investigación Científica Sección Ciencias del mar, UABCS, 6(1), 29-38. [108]

Reyes-Bonilla, H., \& López-Pérez, A. (1998). Biogeografía de los corales pétreos (Scleractinia) del Pacífico de México. Ciencias Marinas, 24(2), 211-224.

Ríos-Jara, E., Galván-Villa, C.M., \& Solís-Marín, F.A. (2008a). Equinodermos del Parque Nacional Isla Isabel, Nayarit, México. Revista Mexicana de Biodiversidad, 79, 131-141. [109]

Ríos-Jara, E., López-Uriarte, E., Pérez-Peña, M., EncisoPadilla, I., Arreola-Robles, J.L., Hermosillo-González, A., \& Galván-Villa, C.M. (2008b). Listados taxonómicos de la biota marina del Parque Nacional Isla Isabel (invertebrados, peces y macroalgas). Zapopan, México: Universidad de Guadalajara. [110]

Ríos-Jara, E., Galván-Villa, C.M., Rodríguez-Zaragoza, F.A., López-Uriarte, E., Bastida-Izaguirre, D., \& Solís-Marín, F.A. (2013). Los equinodermos (Echinodermata) de bahía Chamela, Jalisco, México. Revista Mexicana de Biodiversidad, 84, 263-279. [111]

Ríos-Jara, E., Juárez-Carrillo, E., \& Galván-Villa, C.M. (2017) Invertebrados marinos. In CONABIO (Ed.), La biodiversidad en Jalisco. Estudio de Estado (Vol. 2, pp. 251-269). México: Comisión Nacional para el Conocimiento y Uso de la Biodiversidad, Secretaría de Medio Ambiente y Desarrollo Territorial. [112]

Rodríguez-Troncoso, A.P., Sotelo-Casas, R.C., GalvánVilla, C.M., Godínez-Domínguez, E., Flores-Ortega, J.R., del Castillo-Fernández, K.M., . . .Solís-Marín, F.A. (2013). Equinodermos de la Costa Sur de Jalisco y Colima. In E. Godínez-Domínguez \& M.C. FrancoGordo (Eds.), Inventario de biodiversidad de la costa Sur de Jalisco y Colima, (Vol. 2 pp. 46-60). México: Universidad de Guadalajara. [113]

Roux, M. (2004). New hyocrinid crinoids (Echinodermata) from submersible investigations in the Pacific Ocean. Pacific Science, 58(4), 597-613. [114]

Roux, M., \& Pawson, D.L. (1999). Two new Pacific Ocean species of Hyocrinid crinoids (Echinodermata), with comments on presumed giant-dwarf gradients related to seamounts and abyssal plains. Pacific Science, 53(3), 289-298.

Rowe, F.W.E., \& Pawson, D.L. (1977). A catalogue of echinoderm type-specimens in the Australian Museum, 
Sydney. Records of The Australian Museum, 30(14), 337-364. [115]

Santos-Beltrán, C., \& Salazar-Silva, P. (2011). Holoturoideos (Echinodermata: Holothuroidea) de playas rocosas, zona norte de Bahía de Banderas, Nayarit, México. Ciencia y Mar, 15(45), 3-11. [116]

Sladen, W.P. (1889). Report on the Asteroidea collected by the H. M. S. Challenger during the years 1873-1876. The Voyage of H. M. S. Challenger, Zoology, 30, 1-893. [117]

Solís-Marín, F.A., \& Laguarda-Figueras, A. (1999). Cucumaria flamma, a new species of sea cucumber from the central eastern Pacific (Echinodermata: Holothuroidea). Proceedings of the Biological Society of Washington, 112(4), 778-786. [118]

Solís-Marín, F.A., Arriaga-Ochoa, J.A., Laguarda-Figueras, A., Frontana-Uribe, S.C., \& Durán-González, A. (2009). Holoturoideos (Echinodermata: Holothuroidea) del Golfo de California. México: Comisión Nacional para el Conocimiento y Uso de la Biodiversidad, Instituto de Ciencias del Mar y Limnología, UNAM. [119]

Solís-Marín, F.A., Alvarado, J.J., Abreu-Pérez, M., Aguilera, O., Alió, J., Bacallado-Aránega, J.J., ... Williams, S.M. (2013a). Appendix. In J.J. Alvarado Barrientos \& F.A. Solís-Marín (Eds.), Echinoderm Research and diversity in Latin America (pp. 543-658). Berlin, Germany: Springer-Verlag. [120]

Solís-Marín, F.A., Honey-Escandón, M.B.I., HerreroPérezrul, M.D., Benítez-Villalobos, F., Díaz-Martínez, J.P., Buitrón-Sánchez, B.E., ... Durán-González, A. (2013b). The echinoderms of Mexico: Biodiversity, distribution and current state of knowledge. In J.J. Alvarado \& F.A. Solís-Marín (Eds.), Echinoderm Research and diversity in Latin America (pp. 11-65). Berlin, Germany: Springer-Verlag. [121]

Solís-Marín, F.A., Laguarda-Figueras, A., \& Honey-Escandón, M. (2014). Biodiversidad de equinodermos (Echinodermata) en México. Revista Mexicana de Biodiversidad, 85, S441-S449. [122]

Solís-Marín, F.A., Durán-González, A., \& HernándezHerrejón, L.A. (2016a). Ofiuros (Ophiuroidea). In CONABIO (Ed.), La Biodiversidad en Colima. Estudio de Estado (pp. 305-308). México: Comisión Nacional para el Conocimiento y Uso de la Biodiversidad. [123]

Solís-Marín, F.A., Laguarda-Figueras, A., \& Durán-González, A. (2016b). Estrellas, erizos y pepinos de mar (Echinodermata). Apéndice 1. Lista de equinodermos. In CONABIO (Ed.), La Biodiversidad en Colima. Estudio de Estado (pp. 295-304, 203-205). México: Comisión Nacional para el Conocimiento y Uso de la Biodiversidad. [124]
Solís-Marín, F.A., Arriaga-Ochoa, J.A., Galván-Villa, C.M., \& Laguarda-Figueras, A. (2018). Lissothuria imbricata sp. nov., a new species of sea cucumber (Echinodermata: Holothuroidea) from the centraleastern Pacific Ocean. Zootaxa, 4438(2), 373-380. [125]

Sotelo-Casas, R.C., \& Rodríguez-Troncoso, A.P. (2014). Los equinodermos: invertebrados esenciales dentro de la estructura de la comunidad coralina. In J.L. Cifuentes-Lemus \& F.G. Cupul-Magaña (Coords.), Temas sobre investigaciones costeras (pp. 56-81). México: Universidad de Guadalajara. [126]

Sotelo-Casas, R.C., Cupul-Magaña, A.L., Solís-Marín, F.A., \& Rodríguez-Troncoso, A.P. (2015). New records of sea cucumbers (Echinodermata: Holothuroidea) at Islas Marietas, Central Mexican Pacific. Marine Biological Association of the United Kingdom, 8, 1-8. [127]

Sotelo-Casas, R.C., Cupul-Magaña, A.L., Solís-Marín, F.A., \& Rodríguez-Troncoso, A.P. (2016). Recruitment patterns of 2 sea cucumber species in a Central Mexican Pacific coral reef community. Revista Mexicana de Biodiversidad, 87, 86-91. [128]

Sotelo-Casas, R.C., Cupul-Magaña, A.L., RodríguezZaragoza, F.A., Solís-Marín, F.A., \& RodríguezTroncoso, A.P. (2018). Structural and environmental effects on an assemblage of echinoderms associated with a coral community. Marine Biodiversity, 48, 1401-1411. [129]

Spalding, M.D., Fox, H.E., Allen, G.R., Davidson, N., Ferdaña, Z.A., Finlayson, M., . . . Robertson, J. (2007). Marine ecoregions of the world: A bioregionalization of coastal and shelf areas. BioScience, 57(7), 573-583.

Steinbeck, J., \& Ricketts, E.F. (1941). Sea of Cortez. A leisurely journal of travel and research. New York, USA: Viking Press. [130]

Stöhr, S., O’Hara, T., \& Thuy, B. (2020). World Ophiuroidea database. Retrieved from http://www.marinespecies.org/ophiuroidea

Tommasi, L.R. (1976). Ophiuroidea collected in the PeruChile trench by the USNS "Eltanin" during Cruise III. Papéis Avulsos de Zoologia, 29(8), 281-318. [131]

Valdés de Anda, M., Hendrickx, M.E., \& Solís-Marín, F.A. (2018). New records of Florometra serratissima (A.H. Clark, 1907) (Echinodermata: Crinoidea) from the Gulf of California and the Pacific Coast of Mexico. Cashiers de Biologie Marine, 59, 167-173. [132]

Vergara-Chen, C., Guerra, Z., \& Collado, G.N. (2015). El pepino de mar, Isostichopus fuscus, recurso marino 
en peligro con altas necesidades de manejo. Tecnociencia, 17(2), 21-41. [133]

Villalobos, A.F. (1992). La Isla Socorro. Notas acerca del aspecto hidrobiológico de la parte sur de la Isla. (tomando de Monografías del Instituto de Geofísica 2: 155-180). Hidrobiológica, 2(1-2), 65-91. [134]

WoRMS (2020). World Register of Marine Species. Retrieved from http://www.marinespecies.org/aphia. php? $\mathrm{p}=$ search

Ziesenhenne, F.C. (1937). The Templeton Crocker Expedition. X. Echinoderms from the West Coast of Lower
California, the Gulf of California and Clarion Island. Zoologica, New York Zoological Society, 22(15), 209-239. [135]

Ziesenhenne, F.C. (1940). New Ophiurans of the Allan Hancock Pacific Expeditions. Allan Hancock Pacific Expeditions, 8(2), 9-52. [136]

Ziesenhenne, F.C. (1955). A review of the genus Ophioderma Müller and Troschel. In Essays in the Natural Sciences in honor of Captain Allan Hancock, on the occasion of his birthday, July 26, 1955 (pp. 185-201). Los Angeles, USA: University of Southern California Press. [137]

See Digital Appendix at: / Ver Apéndice digital en: revistas.ucr.ac.cr 